\title{
The correlation consistent composite approach (ccCA): An alternative to the Gaussian- $n$ methods
}

\author{
Nathan J. DeYonker, a) Thomas R. Cundari, and Angela K. Wilson \\ Center for Advanced Scientific Computing and Modeling (CASCaM), Department of Chemistry, \\ The University of North Texas, Denton, Texas 76203
}

(Received 31 October 2005; accepted 19 January 2006; published online 17 March 2006)

\begin{abstract}
An alternative to the Gaussian- $n$ (G1, G2, and G3) composite methods of computing molecular energies is proposed and is named the "correlation consistent composite approach" (ccCA, ccCA-CBS-1, ccCA-CBS-2). This approach uses the correlation consistent polarized valence (cc-pV $X Z)$ basis sets. The G2-1 test set of 48 enthalpies of formation $\left(\Delta H_{f}\right), 38$ adiabatic ionization potentials (IPs), 25 adiabatic electron affinities (EAs), and 8 adiabatic proton affinities (PAs) are computed using this approach, as well as the $\Delta H_{f}$ values of 30 more systems. Equilibrium molecular geometries and vibrational frequencies are obtained using B3LYP density functional theory. When applying the ccCA-CBS method with the cc-pVXZ series of basis sets augmented with diffuse functions, mean absolute deviations within the G2-1 test set compared to experiment are $1.33 \mathrm{kcal} \mathrm{mol}^{-1}$ for $\Delta H_{f}, 0.81 \mathrm{kcal} \mathrm{mol}^{-1}$ for IPs, $1.02 \mathrm{kcal} \mathrm{mol}^{-1}$ for EAs, and $1.51 \mathrm{kcal} \mathrm{mol}^{-1}$ for PAs, without including the "high-level correction" (HLC) contained in the original Gn methods. Whereas the HLC originated in the Gaussian-1 method as an isogyric correction, it evolved into a fitted parameter that minimized the error of the composite methods, eliminating its physical meaning. Recomputing the G1 and G3 enthalpies of formation without the HLC reveals a systematic trend where most $\Delta H_{f}$ values are significantly higher than experimental values. By extrapolating electronic energies to the complete basis set (CBS) limit and adding G3-like corrections for the core-valence and infinite-order electron correlation effects, ccCA-CBS-2 often underestimates the experimental $\Delta H_{f}$, especially for larger systems. This is desired as inclusion of relativistic and atomic spin-orbit effects subsequently improves theoretical $\Delta H_{f}$ values to give a $0.81 \mathrm{kcal} \mathrm{mol}^{-1}$ mean absolute deviation with ccCA-CBS-2. The ccCA-CBS method is a viable "black box" method that can be used on systems with at least 10-15 heavy atoms. (C) 2006 American Institute of Physics. [DOI: $10.1063 / 1.2173988]$
\end{abstract}

\section{INTRODUCTION}

The guiding principle of a "composite method" in $a b$ initio quantum chemistry is straightforward, to mimic the accuracy of an expensive and often computationally intractable electronic structure computation using additive approximations obtained from multiple computations of higher efficiency and lower accuracy. The oldest, most widely used, and arguably most successful composite methods are the Gaussian- $n$ or $\mathrm{G} n$ methods of Pople, Curtiss, and co-workers. ${ }^{1-5}$ The specific goal of these methods was to create a "black box" procedure that could be applied to any (main group) molecular or atomic system and agree with known energetic experimental data [i.e., relative conformational energies, atomization energies, enthalpies of formation $\left(\Delta H_{f}\right)$, ionization potentials (IPs), electron affinities (EAs), and proton affinities (PAs)] to near-chemical accuracy, or within $\pm 2 \mathrm{kcal} \mathrm{mol}^{-1}$. If the composite method achieved this goal over a training set of well-studied chemical species, the same level of accuracy was expected when utilized on species with larger experimental uncertainty or systems where experimental data were unavailable. The $\mathrm{G} n$ methods have

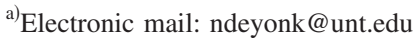

proven to be quite robust in this respect, described in detail within numerous successful applications and reviews. ${ }^{6-10}$ Other composite methods have been created since the original formulation of the G1 method. Some of these, such as the complete basis set (CBS- $n$ ) model chemistries of Petersson and co-workers, ${ }^{11-14}$ the focal point method of Allen and co-workers ${ }^{15-20}$ and Császár et al., ${ }^{21,22}$ the W1 and W2 methods of Parthiban and Martin ${ }^{23}$ and Martin and de Oliveira, ${ }^{24}$ and the High Accuracy Extrapolated $a b$ initio Thermochemistry (HEAT) method of Stanton and co-workers, ${ }^{25,26}$ attempt to approach the complete basis set (CBS)/full-configuration interaction (FCI) limit of smaller systems, consistently obtaining accuracy better than within $0.5 \mathrm{kcal} \mathrm{mol}^{-1}$ of experimental data. Dixon, Feller, and co-workers ${ }^{27-35}$ used large basis set coupled cluster equilibrium geometries and total energies while using smaller basis sets to perform further electron correlation and scalar relativistic corrections, in order to achieve at least chemical accuracy $\left( \pm 1 \mathrm{kcal} \mathrm{mol}^{-1}\right)$ for enthalpies of formation. Feller and Peterson ${ }^{36,37}$ have also examined the performance of G2 methods compared to coupled cluster and perturbation theories. Other model chemistries, such as the multicoefficient correlation method (MCCM) of Truhlar and co-workers, optimize parameters to 
approach the CBS FCI correlation energy. ${ }^{38-40}$ Attempts to parametrize aspects of density functional theory ${ }^{41}$ (DFT) and perturbation theory ${ }^{42}$ (MP2) energy computations to attain accurate enthalpies of formation have also been attempted.

Preferably, a composite method should stay true to the tenets of "first principles" quantum chemistry, where accurate energies are obtained without semiempirical or optimized parameters. As is well known, the $\mathrm{G} n$ methods include such an optimized parameter, the "high-level correction," or HLC. Initially, the HLC of the Gaussian-1 (G1) method had a physical foundation, in that it equaled the difference between the G1 electronic energy of the primary systems (hydrogen atom and hydrogen molecule) and their near-exact analytical energies. ${ }^{3}$ It was then assumed that any unpaired valence electrons in a molecular system would also need a HLC equivalent to that of the $\mathrm{H}$ atom, and likewise that each pair of electrons would require the same $\mathrm{HLC}$ as the $\mathrm{H}_{2}$ molecule. Thus within the G1 method, the HLC acted as an isogyric correction.

The simple physical implications of the G1 HLC were abandoned upon the advent of the Gaussian-2 (G2) method. ${ }^{4}$ The G2 HLC was optimized in order to minimize the mean absolute deviation from experiment for the 55 atomization energies investigated in the initial G2-1 training set. Henceforth, the HLC became a semiempirical parameter somewhat in opposition to the spirit of $a b$ initio electronic structure methods. It is not well known if the HLC is needed because of the use of an incomplete basis set, incomplete treatment of electron correlation, the use of the harmonic approximation to compute vibrational zero-point energies, bond additivity effects, or even perhaps a combination of these effects. There are also smaller contributions to the electronic energy such as spin-orbit splitting, and relativistic effects that influence the HLC. To our knowledge, no work has been carried out to discern how these effects interplay with the magnitude of the HLC, nor has there been a focus on how $\mathrm{G} n$ methods fare without inclusion of the HLC.

In 1989, the same year that the G1 method was published, Dunning ${ }^{43}$ first introduced correlation consistent polarized valence (cc-pVXZ with $X=D, T, Q, 5, \ldots$ ) basis sets for the first-row atoms. The correlation consistent basis sets were constructed by systematically expanding the higher angular momentum functions in shells. These basis sets were found to result in convergence toward the one-particle CBS limit for a number of properties for a given correlated method. $^{43-45}$ The systematic nature of this convergence has enabled energies of increasing basis set size to be used to extrapolate to the CBS limit. By extrapolating to the CBS limit, the error coupling of the one-particle basis set expansion and the $n$-particle electron correlation is eliminated, leaving only the intrinsic error of the trial electronic wave function. Correlation consistent basis sets have since been reported for the second- and third-row elements, ${ }^{45-47}$ and have been modified to describe molecular systems where electron density is diffuse (aug-cc-pVXZ) (Refs. 48 and 49) or to account for core-valence electron correlation (cc-pCV $X Z){ }^{50,51}$ Most recently, cc-pVXZ and their augmented and core-valence versions have been constructed for the $3 d$ transition metals by Balabanov and Peterson. ${ }^{52}$
With respect to basis set size, the poor scaling of $a b$ initio theories that can account for high levels of electron correlation remains the limiting factor in the maximum molecular size that can be addressed by a composite method. Dixon and co-workers ${ }^{34,35}$ have demonstrated that employing quadruple-zeta (the minimum "zeta level" typically necessary for satisfactory CBS extrapolations) coupled cluster computations on molecules of modest size (e.g., n-octane) require extraordinary computational resources. Additionally, accounting for smaller, but significant energy corrections such as higher-order electron correlation, relativistic effects, spin-orbit coupling, and anharmonic zero-point energies can be time consuming and require more extensive expertise and software. These aspects prohibit the realization of a flexible "black box" composite method untethered by empirical or optimized parameters, that is also applicable to large molecules.

While composite methods utilizing cc-pVXZ basis sets and CBS extrapolation schemes exist, to our knowledge, no thorough attempt has been made to combine cc-pVXZ basis sets with the time-saving aspects of the $\mathrm{G} n$ model chemistries. In this paper, the "Dunning-style" cc-pVXZ basis sets will replace the "Pople-style" basis sets traditionally used in the $\mathrm{G} n$ methods. If the larger and more flexible cc-pVXZ families of basis set are used as the reference for the various $\mathrm{G} n$ additive corrections, near-chemical accuracy of $\pm 2 \mathrm{kcal} \mathrm{mol}^{-1}$ can be achieved for properties on a variety of chemical systems. Our modifications to the $\mathrm{G} n$ methods, called the "correlation consistent composite approach" or ccCA and ccCA-CBS were designed to include no semiempirical or optimized parameters. The ccCA-CBS methods could certainly be considered a more efficient approximation to the composite method of Dixon and co-workers, via substitution of coupled cluster geometry optimizations with perturbation theory single point energies applied to DFT equilibrium geometries. The ccCA and ccCA-CBS energies will be presented for 118 of the 125 atomic and molecular systems contained in the G2-1 subset, ${ }^{4}$ a selection of systems from the G2/97 (Ref. 6), and G3/99 (Ref. 8) test sets, and two large systems from the 600-molecule training set proposed by Cioslowski et al. ${ }^{41}$ The performance of the ccCA will be examined and compared to experiment, $\mathrm{G} n$ methods (with and without their respective HLCs), and other theoretical studies.

\section{DESCRIPTION OF THE CCCA THEORETICAL METHODS}

$\mathrm{G} n$ composite methods have been described in detail in Refs. 2-11, as well as references therein. They comprise of a set of $a b$ initio molecular orbital (MO)-based computations performed to give an equilibrium geometry, a set of harmonic vibrational frequencies, and a total electronic energy at the equilibrium geometry. The determination of G3 geometries, frequencies, and total energies are described as follows.

To obtain the G3 equilibrium structure, a second-order Møller-Plesset perturbation theory ${ }^{53,54}$ (MP2) computation is run at the $6-31 \mathrm{G}(d)$ basis set level ${ }^{55}$ with all electrons corre- 
lated. Harmonic vibrational frequencies at $0 \mathrm{~K}$ are computed earlier, using the global minimum obtained at the HF/6-31 $\mathrm{G}(d)$ level of theory and scaled by a factor of 0.8929 to give the zero-point energy $[E(\mathrm{ZPE})]$.

In G3, a reference energy is computed with complete fourth-order Møller-Plesset perturbation theory ${ }^{56}$ and the 6-31G $(d)$ basis set at the MP2(full)/6-31G $(d)$ equilibrium geometry. [In G1 and G2, the reference energy is computed at the MP4/6-311G $(d, p)$ level.] Shown here for G3, additive single point energy corrections are obtained with the following additional computations.

(a) $\Delta E(+)$, a MP4 computation where the $6-31 \mathrm{G}(d)$ basis set is augmented with diffuse functions, $6-31+\mathrm{G}(d){ }^{57,58}$

(b) $\Delta E(2 d f, p)$, a MP4 computation where higher polarization functions are added to the $6-31 \mathrm{G}(d)$ basis set, 6-31G $(2 d f, p)$.

(c) $\Delta E(\mathrm{QCI})$, a correction to include correlation effects to infinite-order using quadratic configuration interaction with single, double, and perturbative triple excitations [QCISD(T)] (Ref. 59) with the 6-31G $(d)$ basis set.

(d) In the G3 method, an all-electron MP2 correction is included $[\Delta E$ (G3large) $]$ with an even larger basis set to consider core-valence correlation effects.

(e) In the G3 method, atomic spin-orbit corrections are taken from experimental results ${ }^{60}$ or theoretical computations. ${ }^{5}$

(f) Lastly, the HLC is added depending on the number of closed- and open-shell valence electrons. The actual value of the HLC for each open-shell electron or bound pair of closed shell electrons differs amongst the $\mathrm{G} n$ methods.

Effectively for G1 and G2 methods the result of these additive corrections to the MP4/6-311G(d,p) // MP2(full)/ 6-31G $(d)$ total energy should be equal to the zero-point corrected $\left(E_{0}\right)$ QCISD(T)/6-311+G(2df, $\left.p\right)$ and $\operatorname{QCISD}(\mathrm{T}) / 6$ $311+\mathrm{G}(3 d f, 2 p)$ energies, respectively, while for $\mathrm{G} 3$, the composite method should give the $\mathrm{E}_{0}$ at the $\operatorname{QCISD}(\mathrm{T}, \mathrm{full}) /$ G3large level of theory. The validity of using the $\mathrm{G} n$ composite methods to reach the target total energy of the higher level of theory has been found to be satisfactory. ${ }^{61}$

Many modifications to the $\mathrm{G} n$ methods have appeared such as reducing the basis set size, ${ }^{62}$ employing coupled cluster with single, double, and perturbative triple excitations $[\operatorname{CCSD}(\mathrm{T})]$ (Refs. 59 and 63) instead of QCISD(T), using second- or third-order MPn theory instead of MP4 to determine the reference electronic energy, ${ }^{64,65}$ and using scale factors to replace the HLC. ${ }^{66,67}$ The ccCA will adopt the use of B3LYP DFT equilibrium geometries and vibrational frequencies similar to the modifications of the G3 methods called G3/B3LYP (or G3B3) (Ref. 68) and G3X. ${ }^{67}$ This variation generally retains sufficient accuracy while severely reducing the computational expense of geometry optimizations. ${ }^{68,69}$

The first implementation of ccCA attempts to remain similar to the G3B3 algorithm due to the demonstrated success of the G3 approaches. Our main modification is based on the relationship between basis set size and correlation energy in attaining accurate theoretical thermochemical values and relative energies. As basis sets and electron correlation effects are the two largest components which determine the accuracy of computing atomic and molecule electronic wave functions, these effects must be the focus of a composite $a b$ initio technique. While the Gaussian- $n$ methods account for a significant portion of the dynamical electron correlation with the MP4 level of theory, the additive corrections may not be balanced if employed in conjunction with rather small basis sets. This investigation will show that without an empirical or parametrized energy correction, small basis sets such as 6-31G $(d)$ or cc-pVDZ are of insufficient quality to act as the reference electronic energy for additive corrections since the correlated methods used [MP2, MP4, QCISD(T)] are heavily basis set dependent. It will be demonstrated that if larger or CBS-extrapolated correlation consistent basis sets are employed as the foundation for additive corrections, the accuracy of ccCA improves dramatically. The algorithms of ccCA are outlined as follows.

The equilibrium geometries are obtained at the B3LYP/ 6-31G $(d)$ level of theory (akin to G3B3 theory) ${ }^{6}$ and harmonic frequencies are also computed at the B3LYP/6-31 $\mathrm{G}(d)$ level of theory and scaled by a factor of 0.9854 (equivalent to the scale factor used in G3X) ) $^{67,70,71}$ in order to compute the zero-point vibrational energy correction at $0 \mathrm{~K}$ and the molecular enthalpy of formation correction at 298.15 $\mathrm{K}$. The G3X scale factor (with G3B3 geometries) is used, which generally improves ccCA-derived enthalpies of formation by $0.10 \mathrm{kcal} \mathrm{mol}^{-1}$ as compared to the G3B3 scale factor of 0.96. Again, the main impetus behind using the DFT geometries and harmonic ZPE corrections is that there is relatively little compromise in accuracy compared to the time savings achieved from avoiding expensive MP2 or $\operatorname{CCSD}(\mathrm{T})$ geometry optimizations. Geometry optimizations at the $\operatorname{CCSD}(\mathrm{T})$ level of theory are still prohibitively expensive for most large molecules, and efficient parallelized frozen-core coupled cluster energy gradients are nonexistent in most widely available ab initio software packages. Variation of ccCA accuracy based on different types of optimized geometries will be further investigated in future work, though we believe that the 6-31G $(d)$ DFT geometries with the G3X zero-point energy scheme will be important for a successful "black box" implementation on large molecular systems.

We have initially implemented five variants of the ccCA methods. Three versions of ccCA begin with a single point frozen-core MP4 computation along with a correlation consistent basis set used as the reference energy at the B3LYP/ 6-31G $(d)$ equilibrium geometry for subsequent additive corrections. If the reference energy is computed with the ccpVDZ basis sets, the method is named ccCA-DZ. Likewise, with a cc-pVTZ reference energy the method is called ccCA$\mathrm{TZ}$, and an aug-cc-pVTZ reference energy is called ccCAaTZ. Additive corrections are modeled from the original $\mathrm{G} n$ methods. For example, if MP4/cc-pVTZ is the reference energy (as with ccCA-TZ), $\Delta E(+)$ is computed to correct for the addition of diffuse functions, 


$$
\Delta E(+)=E[\mathrm{MP} 4 / \text { aug-cc-pVTZ }]-E[\mathrm{MP} 4 / \mathrm{cc}-\mathrm{pVTZ}] .
$$

The correction for higher polarization functions can be simply formulated with the cc-pVXZ basis sets by raising the "zeta level," i.e., increasing from double-zeta to triple-zeta, or triple-zeta to quadruple-zeta level basis sets. This was designated as $\Delta E(2 d f, p)$ in $\mathrm{G} n$ methods, and is now named $\Delta E(\zeta)$.

For the latter two variations of the ccCA methods (named ccCA-CBS), frozen-core MP2 energies are computed with the aug-cc-pVDZ, aug-cc-pVTZ, and aug-ccpVQZ basis sets, and extrapolated to the CBS limit. We determine the MP2 CBS energy in two ways, the first is the simple exponential form proposed by Xantheas and Dunning ${ }^{44}$ and Feller, ${ }^{72,73}$ where $x=$ the zeta level of the correlation consistent basis set $[2=\mathrm{DZ}, 3=\mathrm{TZ}$, etc.]:

$$
E(X)=A_{\mathrm{CBS}}+B \exp (-C x) .
$$

Alternatively, a mixed exponential/Gaussian functional devised by Peterson $e t a l .^{74}$ is expressed as

$$
E(x)=A_{\mathrm{CBS}}+B \exp [-(x-1)]+C \exp \left[-(x-1)^{2}\right] .
$$

The ccCA energies determined with these two functional forms are referred to as ccCA-CBS- 1 and ccCA-CBS-2, respectively. This MP2 CBS energy serves as the reference energy for additive corrections.

In order to properly account for high-order electron correlation effects, a small basis set single point energy is computed with the QCISD(T) wave function. If MP2 or MP4 levels of theory overestimate the amount of dynamical correlation in the system, the $\Delta E(\mathrm{QCI})$ correction can actually raise the total energy of the system. The $\Delta E(\mathrm{QCI})$ correction for the ccCA-aTZ method is expressed as

$$
\begin{aligned}
\Delta E(\mathrm{QCI})= & E[\mathrm{QCISD}(\mathrm{T}) / \mathrm{cc}-\mathrm{pVTZ}] \\
& -E[\mathrm{MP} 4 / \mathrm{cc}-\mathrm{pVTZ}] .
\end{aligned}
$$

Within the ccCA-CBS methods $\Delta E(\mathrm{QCI})$ is

$$
\begin{aligned}
\Delta E(\mathrm{QCI})= & E[\mathrm{QCISD}(\mathrm{T}) / \mathrm{cc}-\mathrm{pVTZ}] \\
& -E[\mathrm{MP} 2 / \mathrm{cc}-\mathrm{pVTZ}] .
\end{aligned}
$$

The G3large correction is implemented as a simple correction for core-valence correlation effects and renamed $\Delta E(\mathrm{CV})$. For the ccCA methods with triple-zeta reference energies, $\Delta E(\mathrm{CV})$ is computed as

$$
\begin{aligned}
\Delta E(\mathrm{CV})= & E[\mathrm{MP} 2(\text { full }) / \text { aug-cc-pCVTZ }] \\
& -E[\mathrm{MP} 2 / \text { aug-cc-pVTZ }] .
\end{aligned}
$$

All of the appropriate additive corrections, such as the zeropoint energy correction $[\Delta E(\mathrm{ZPE})], \Delta \mathrm{E}(+), \Delta \mathrm{E}(\zeta), \Delta E(\mathrm{QCI})$, and $\Delta E(\mathrm{CV})$, are added to the reference energy. Finally, depending on the basis set used to compute the reference energy, the ccCA energy is defined as

$$
\begin{aligned}
E_{0}(\mathrm{ccCA}-\mathrm{DZ})= & E[\mathrm{MP} 4 / \mathrm{cc}-\mathrm{pVDZ}]+\Delta E(+)+\Delta E(\zeta) \\
& +\Delta E(\mathrm{QCI})+\Delta E(\mathrm{CV})+\Delta E(\mathrm{ZPE}),
\end{aligned}
$$

$$
\begin{aligned}
E_{0}(\mathrm{ccCA}-\mathrm{TZ})= & E[\mathrm{MP} 4 / \mathrm{cc}-\mathrm{pVTZ}]+\Delta E(+)+\Delta E(\zeta) \\
& +\Delta E(\mathrm{QCI})+\Delta E(\mathrm{CV})+\Delta E(\mathrm{ZPE})
\end{aligned}
$$

or

$$
\begin{aligned}
E_{0}(\mathrm{ccCA}-\mathrm{aTZ})= & E[\mathrm{MP} 4 / \text { aug-cc-pVTZ }]+\Delta E(\zeta) \\
& +\Delta E(\mathrm{QCI})+\Delta E(\mathrm{CV})+\Delta E(\mathrm{ZPE})
\end{aligned}
$$

Both of the ccCA-CBS energies are defined as

$$
\begin{aligned}
E_{0}(\mathrm{ccCA}-\mathrm{CBS}-n)= & E[\mathrm{MP} 2 / \text { aug }-\mathrm{cc}-\mathrm{pV} \infty \mathrm{Z}]+\Delta E(\mathrm{QCI}) \\
& +\Delta E(\mathrm{CV})+\Delta E(\mathrm{ZPE}) .
\end{aligned}
$$

Table I pictorially represents the additive corrections used with the ccCA method and a direct comparison to the $\mathrm{G} n$ algorithms. All computations were run with the GAUSSIAN03 software package ${ }^{75}$ except for the $n$-octane, $\mathrm{C}\left(\mathrm{NO}_{2}\right)_{4}$, and adamantane QCISD(T) computations, for which MOLPRO 2002.6 was used. ${ }^{76}$ Closed shell restricted Hartree-Fock (RHF) wave functions and open-shell unrestricted HartreeFock (UHF) reference wave functions were implemented. Newly optimized (aug)-cc-p(C)VXZ basis sets for $\mathrm{Li}, \mathrm{Be}$, $\mathrm{Mg}$, and $\mathrm{Na}$ atoms were obtained from Peterson et al. ${ }^{77}$

\section{BASIS SET DEPENDENCE OF REFERENCE ENERGIES}

In this study, a major goal is to examine the role of the initial electronic energy to be used as the foundation for additive corrections. The most crucial difference between the $\mathrm{G} n$ composite methods and the ccCAs is that the ccCA basis set sizes are generally larger. To make a brief comparison of increasing the basis set size for the reference energy, atomic energies are listed in Table II obtained with the $\mathrm{G} n$ methods, those utilizing cc-pVDZ (ccCA-DZ), cc-pVTZ (ccCA-TZ), and aug-cc-pVTZ (ccCA-aTZ) basis sets as the reference MP4 energy, and the ccCA-CBS- 1 and ccCA-CBS-2 energies. The first point of comparison is between the G3 atomic energies and those obtained with the ccCA-DZ method. Of the non-noble gas first-row atomic electronic energies, the ccCA-DZ energies are higher (and none more than $10 \mathrm{~m} E_{\mathrm{h}}$ ) than the G3 energies, while the second-row ccCA-DZ energies are usually higher by $80-90 \mathrm{~m} E_{\mathrm{h}}$. However, the G3 energies include the HLC, which can contribute up to $24.56 \mathrm{~m} E_{\mathrm{h}}$ to the electronic energy for the first- and secondrow atoms. All computed ccCA-TZ, ccCA-aTZ, and ccCACBS atomic energies are significantly lower than the G3 energies, except for the hydrogen radical, which G3 predicts to violate the variational principle due to overcorrection of the parametrized HLC with an electronic energy of $-0.501 E_{\mathrm{h}}$. Likewise, the hydrogen atom energy predicted with the ccCA-CBS-2 composite method is below the variational limit, in this case by $20 \mu E_{\mathrm{h}}$. While none of these model chemistries strictly adhere to the variational principle, the recovery of more atomic correlation energy due to larger basis set size in the ccCA methods is an early indicator that molecular properties will achieve comparable or improved accuracy when compared to the $\mathrm{G} n$ methods.

To examine the basis set dependence, Table III shows atomic IPs and EAs obtained with the $\mathrm{G} n$ and ccCA meth- 
TABLE I. Depiction of the $\mathrm{G} n$ and ccCA algorithms.

\begin{tabular}{|c|c|c|c|c|c|c|c|}
\hline & $\mathrm{G} 1^{\mathrm{a}}$ & $\mathrm{G} 2^{\mathrm{b}}$ & $\mathrm{G} 3 \mathrm{~B} 3^{\mathrm{c}}$ & ccCA-DZ & ccCA-TZ & ccCA-aTZ & ccCA CBS- 1 / ccCA CBS-2 \\
\hline $\begin{array}{l}\text { Geometry } \\
\text { optimization }\end{array}$ & MP2(full)/6-311G ${ }^{* *}$ & MP2(full)/6-31G(d) & B3LYP/6-31G $(d)$ & B3LYP/6-31G $(d)$ & B3LYP/6-31G $(d)$ & B3LYP/6-31G $(d)$ & B3LYP/6-31G $(d)$ \\
\hline $\begin{array}{l}\text { Harmonic } \\
\text { vibrational } \\
\text { frequencies }\end{array}$ & $\mathrm{HF} / 6-31 \mathrm{G}(d)$ & $\mathrm{HF} / 6-31 \mathrm{G}(d)$ & B3LYP/6-31G $(d)$ & B3LYP/6-31G $(d)$ & B3LYP/6-31G $(d)$ & B3LYP/6-31G $(d)$ & B3LYP/6-31G $(d)$ \\
\hline Scale factor & 0.8929 & 0.8929 & 0.9854 & 0.9854 & 0.9854 & 0.9854 & 0.9854 \\
\hline $\begin{array}{l}\text { Reference energy } \\
E(\mathrm{ref})\end{array}$ & $\mathrm{MP} 4 / 6-311 \mathrm{G}^{* *}$ & $\mathrm{MP} 4 / 6-311 \mathrm{G}(d, p)$ & $\mathrm{MP} 4 / 6-31 \mathrm{G}(d)$ & MP4/cc-pVDZ & MP4/cc-pVTZ & MP4/aug-cc-pVTZ & MP2/CBS aug-cc-pVXZ \\
\hline$\Delta E(+)$ & $\begin{array}{c}\mathrm{MP} 4 / 6-311+\mathrm{G}^{* *} \\
-E(\mathrm{ref})\end{array}$ & $\begin{array}{c}\mathrm{MP} 4 / 6-311+\mathrm{G}(d, p) \\
-E(\mathrm{ref})\end{array}$ & $\begin{array}{c}\mathrm{MP} 4 / 6-31+\mathrm{G}(d) \\
-E(\mathrm{ref})\end{array}$ & $\begin{array}{c}\mathrm{MP} 4 / \text { aug-cc-pVDZ } \\
-E \text { (ref) }\end{array}$ & $\begin{array}{c}\text { MP4/aug-cc-pVTZ } \\
-E \text { (ref) }\end{array}$ & $\ldots$ & $\ldots$ \\
\hline$\Delta E(2 d f, p) / \Delta E(\zeta)$ & $\begin{array}{c}\mathrm{MP} / 6-311 \mathrm{G}^{* *}(2 d f) \\
-E(\mathrm{ref})\end{array}$ & $\begin{array}{c}\mathrm{MP} 4 / 6-311 \mathrm{G}(2 d f, p) \\
-E(\mathrm{ref})\end{array}$ & $\begin{array}{c}\mathrm{MP} 4 / 6-31 \mathrm{G}(2 d f, p) \\
-E(\mathrm{ref})\end{array}$ & $\begin{array}{l}\mathrm{MP} 4 / \mathrm{cc}-\mathrm{pVTZ} \\
-E(\mathrm{ref})\end{array}$ & $\begin{array}{c}\text { MP4/cc-pVQZ } \\
\text {-MP4/ } \\
\text { cc-pVTZ }\end{array}$ & $\begin{array}{l}\text { MP2/aug-cc-pVQZ } \\
\text {-MP2/aug-cc-pVTZ }\end{array}$ & $\cdots$ \\
\hline$\Delta E(\mathrm{QCI})$ & $\mathrm{QCISD}(\mathrm{T}) / 6-311 \mathrm{G}^{* *}-E(\mathrm{ref})$ & $\begin{array}{c}\mathrm{QCISD}(\mathrm{T}) / 6-311 \mathrm{AG}(d, p) \\
-E(\mathrm{ref})\end{array}$ & $\begin{array}{c}\mathrm{QCISD}(\mathrm{T}) / 6-31 \mathrm{G}(d) \\
-E(\mathrm{ref})\end{array}$ & $\begin{array}{c}\mathrm{QCISD}(\mathrm{T}) / \mathrm{cc}-\mathrm{pVDZ} \\
-E(\mathrm{ref})\end{array}$ & $\begin{array}{c}\mathrm{QCISD}(\mathrm{T}) / \mathrm{cc}-\mathrm{pVTZ} \\
-E(\mathrm{ref})\end{array}$ & $\begin{array}{l}\text { QCISD(T)/cc-pVTZ } \\
-\mathrm{MP} 4 / \mathrm{cc}-\mathrm{pVTZ}\end{array}$ & $\begin{array}{l}\mathrm{QCISD}(\mathrm{T}) / \mathrm{cc}-\mathrm{pVTZ} \\
-\mathrm{MP} 2 / \mathrm{cc}-\mathrm{pVTZ}\end{array}$ \\
\hline $\begin{array}{c}\Delta E(\mathrm{G} 3 \text { large }) / \\
\Delta E(\mathrm{CV})\end{array}$ & $\cdots$ & $\begin{array}{c}\mathrm{MP} 2 / 6-311+\mathrm{G}(3 d f, 2 p) \\
-\mathrm{MP} 2 / 6-311 \mathrm{G}(2 d f, p) \\
-\mathrm{MP} 2 / 6-311+\mathrm{G}(d, p) \\
\text { +MP2/6-311G }(d, p)\end{array}$ & $\begin{array}{l}\text { MP2(full)/G3large } \\
-\mathrm{MP} 2 / 6-31 \mathrm{G}(2 d f, p) \\
-\mathrm{MP} 2 / 6-31 \mathrm{G}(2 d f, p) \\
\text {-MP2/6-31+G(d) } \\
\text { +MP2/6-31+G(d) }\end{array}$ & $\begin{array}{c}\text { MP2(full)/ } \\
\text { aug-cc-pCVDZ } \\
\text {-MP2/aug-cc-pVTZ }\end{array}$ & $\begin{array}{c}\text { MP2(full)/ } \\
\text { aug-cc-pCVTZ } \\
\text {-MP2/aug-cc-pVTZ }\end{array}$ & $\begin{array}{c}\text { MP2(full)/ } \\
\text { aug-cc-pCVTZ } \\
\text {-MP2/aug-cc-pVTZ }\end{array}$ & $\begin{array}{l}\text { MP2(full)/aug-cc-pCVTZ } \\
\text {-MP2/aug-cc-pVTZ }\end{array}$ \\
\hline$\Delta E(\mathrm{HLC})$ & $-0.19 n_{\alpha}-5.95 n_{\beta}{ }^{\mathrm{d}}$ & $-0.19 n_{\alpha}-4.81 n_{\beta}^{\mathrm{d}}$ & $-\mathrm{A} n_{\alpha}-\mathrm{B} n_{\beta}{ }^{\mathrm{e}}$ & $\cdots$ & $\cdots$ & $\cdots$ & $\cdots$ \\
\hline $\begin{array}{l}\text { Effective level of } \\
\text { theory }\end{array}$ & $\begin{array}{c}\mathrm{QCISD}(\mathrm{T}) / \\
6-311+\mathrm{G}^{* *}(2 d f)\end{array}$ & $\begin{array}{c}\operatorname{QCISD}(\mathrm{T}) / \\
6-311+\mathrm{G}(3 d f, 2 p)\end{array}$ & $\begin{array}{l}\text { QCISD(T,full)/ } \\
\text { G3large }\end{array}$ & $\begin{array}{l}\text { QCISD(T,full)/ } \\
\text { aug-cc-pCVTZ }\end{array}$ & $\begin{array}{l}\text { QCISD(T,full)/ } \\
\text { aug-cc-pCVQZ }\end{array}$ & $\begin{array}{l}\text { QCISD(T,full)/ } \\
\text { aug-cc-pCVQZ }\end{array}$ & $\begin{array}{l}\text { QCISD(T,full)/aug-cc- } \\
\text { pCVœZ }\end{array}$ \\
\hline \multicolumn{8}{|c|}{$\begin{array}{l}{ }^{\mathrm{a}} \text { Reference } 3 . \\
\text { beference } 4 \text {. The G3 variants also include a spin-orbit correction to atomic energies. } \\
{ }^{\mathrm{c}} \text { Refef. } 68 \text {. } \\
{ }^{\mathrm{d}} n_{\alpha} \text { and } n_{\beta} \text { represent the number of alpha- and beta-spin valence electrons, respectively. } \\
{ }^{\mathrm{e}} \text { The high-level correction parameter } A \text { equals } 6.760 \mathrm{~m} E_{\mathrm{h}} \text { for molecules and } 6.786 \mathrm{~m} E_{\mathrm{h}}\end{array}$} \\
\hline
\end{tabular}


TABLE II. Atomic energies (in $E_{\mathrm{h}}$ ) using $\mathrm{G} n$ methods.

\begin{tabular}{|c|c|c|c|c|c|c|c|}
\hline & G1 & G3 & ccCA-DZ & ccCA-TZ & ccCA-aTZ & ccCA-CBS-1 & ccCA-CBS-2 \\
\hline $\mathrm{H}\left({ }^{2} S\right)$ & -0.50000 & -0.50100 & -0.49987 & -0.49996 & -0.49995 & -0.49999 & -0.50002 \\
\hline $\operatorname{Li}\left({ }^{2} S\right)$ & -7.43222 & -7.46513 & -7.46181 & -7.47023 & -7.47023 & -7.47023 & -7.47023 \\
\hline $\operatorname{Be}\left({ }^{1} S\right)$ & -14.62337 & -14.65972 & -14.65037 & -14.66008 & -14.66090 & -14.66202 & -14.66184 \\
\hline $\mathrm{B}\left({ }^{2} P\right)$ & -24.60279 & -24.64257 & -24.63338 & -24.64531 & -24.64627 & -24.64893 & -24.64857 \\
\hline $\mathrm{C}\left({ }^{3} P\right)$ & -37.78464 & -37.82772 & -37.82036 & -37.83493 & -37.83553 & -37.83902 & -37.83928 \\
\hline $\mathrm{N}\left({ }^{4} S\right)$ & -54.51776 & -54.56434 & -54.55973 & -54.57799 & -54.57780 & -54.58241 & -54.58334 \\
\hline $\mathrm{O}\left({ }^{3} P\right)$ & -74.98204 & -75.03099 & -75.02808 & -75.05385 & -75.05175 & -75.06002 & -75.06130 \\
\hline $\mathrm{F}\left({ }^{2} P\right)$ & -99.63275 & -99.68421 & -99.68389 & -99.71800 & -99.71379 & -99.72644 & -99.72804 \\
\hline $\mathrm{Na}\left({ }^{2} S\right)$ & -161.84618 & -162.10415 & -162.04756 & -162.14760 & -162.14760 & -162.14771 & -162.14788 \\
\hline $\operatorname{Mg}\left({ }^{1} S\right)$ & -199.64613 & -199.90742 & -199.82032 & -166.94885 & -199.94945 & -199.95012 & -199.95049 \\
\hline $\mathrm{Al}\left({ }^{2} P\right)$ & -241.93167 & -242.20747 & -242.13319 & -242.24741 & -242.24830 & -242.24994 & -242.25025 \\
\hline $\operatorname{Si}\left({ }^{3} P\right)$ & -288.93378 & -289.22227 & -289.14136 & -289.25794 & -289.25882 & -289.26148 & -289.26188 \\
\hline $\mathrm{P}\left({ }^{4} S\right)$ & -340.81800 & -341.11643 & -341.03585 & -341.15422 & -341.15484 & -341.15814 & -341.15901 \\
\hline $\mathrm{S}\left({ }^{3} P\right)$ & -397.65493 & -397.96111 & -397.87659 & -398.00163 & -398.00061 & -398.00629 & -398.00758 \\
\hline $\mathrm{Cl}\left({ }^{2} P\right)$ & -459.67670 & -459.99096 & -459.90273 & -460.03821 & -460.03508 & -460.04168 & -460.04479 \\
\hline $\mathrm{Li}^{+}\left({ }^{1} S\right)$ & -7.23584 & 7.26679 & -7.26496 & -7.27273 & -7.27273 & -7.27273 & -7.27272 \\
\hline $\mathrm{Be}^{+}\left({ }^{2} S\right)$ & -14.27639 & -14.31214 & -14.30941 & -14.31715 & -14.31714 & -14.31716 & -14.31719 \\
\hline $\mathrm{B}^{+}\left({ }^{1} S\right)$ & -24.30175 & -24.34000 & -24.33153 & -24.34249 & -24.34324 & -24.34642 & -24.34498 \\
\hline $\mathrm{C}^{+}\left({ }^{2} P\right)$ & -37.37405 & -37.41571 & -37.40750 & -37.42231 & -37.42305 & -37.42633 & -37.42623 \\
\hline $\mathrm{N}^{+}\left({ }^{3} P\right)$ & -53.98600 & -54.03123 & -54.02375 & -54.04364 & -54.04425 & -54.04869 & -54.04935 \\
\hline $\mathrm{O}^{+}\left({ }^{4} S\right)$ & -74.48439 & -74.53312 & -74.52768 & -74.554 .06 & -74.55433 & -74.56088 & -74.56227 \\
\hline $\mathrm{F}^{+}\left({ }^{3} P\right)$ & -98.99362 & -99.04519 & -99.03848 & -99.07641 & -99.07553 & -99.08623 & -99.08839 \\
\hline $\mathrm{Na}^{+}\left({ }^{1} S\right)$ & -161.66423 & -161.91623 & -161.86364 & -161.96135 & -161.96065 & -161.96146 & -161.96165 \\
\hline $\mathrm{Mg}^{+}\left({ }^{2} S\right)$ & -199.36402 & -199.62131 & -199.54182 & -199.66794 & -199.66793 & -199.66812 & -199.68837 \\
\hline $\mathrm{Al}^{+}\left({ }^{1} S\right)$ & -241.71379 & -241.98847 & -241.91443 & -242.02872 & -242.02938 & -242.03034 & -242.03066 \\
\hline $\mathrm{Si}^{+}\left({ }^{2} P\right)$ & -288.63626 & -288.92362 & -288.84140 & -288.95883 & -288.95966 & -288.96202 & -288.96224 \\
\hline $\mathrm{P}^{+}\left({ }^{3} P\right)$ & -340.43437 & -340.73190 & -340.64792 & -340.76760 & -340.76851 & -340.77157 & -340.77223 \\
\hline $\mathrm{S}^{+}\left({ }^{4} S\right)$ & -397.27956 & -397.58373 & -397.49897 & -397.62310 & -397.62376 & -397.62764 & -397.62897 \\
\hline $\mathrm{Cl}^{+}\left({ }^{3} P\right)$ & -459.20384 & -459.51725 & -459.42744 & -459.561 97 & -459.56111 & -459.56733 & -459.56933 \\
\hline $\mathrm{C}^{-}\left({ }^{4} S\right)$ & -37.82825 & -37.87158 & -37.88370 & -37.88880 & -37.88076 & -37.88346 & -37.88391 \\
\hline $\mathrm{O}^{-}\left({ }^{2} P\right)$ & -75.03385 & -75.08014 & -75.11811 & -75.12584 & -75.10547 & -75.11384 & -75.11463 \\
\hline $\mathrm{F}^{-}\left({ }^{1} S\right)$ & -99.75993 & -99.80919 & -99.85780 & -99.86941 & -99.84414 & -99.85585 & -99.85654 \\
\hline $\mathrm{Si}^{-}\left({ }^{4} S\right)$ & -288.98227 & -289.27290 & -289.20080 & -289.31723 & -289.31023 & -289.31260 & -289.31319 \\
\hline $\mathrm{P}^{-}\left({ }^{3} P\right)$ & -340.84018 & -341.14370 & -341.07790 & -341.19026 & -341.17973 & -341.18392 & -341.18484 \\
\hline $\mathrm{S}^{-}\left({ }^{2} P\right)$ & -397.72683 & -398.03701 & -397.97026 & -398.08554 & -398.07537 & -398.08295 & -398.08358 \\
\hline $\mathrm{Cl}^{-}\left({ }^{1} S\right)$ & -459.80793 & -460.12360 & -460.05232 & -460.18031 & -460.16783 & -460.17967 & -460.17947 \\
\hline
\end{tabular}

ods. For the 14 atomic IPs, the accuracy improves when comparing ccCA-DZ and ccCA-TZ, as the average absolute deviation is reduced from 1.34 to $0.64 \mathrm{kcal} \mathrm{mol}^{-1}$, compared to the G3 average absolute deviation of $1.24 \mathrm{kcal} \mathrm{mol}^{-1}$. The ccCA-aTZ method performs slightly worse than other methods for atomic ionization potentials $\left(1.01 \mathrm{kcal} \mathrm{mol}^{-1}\right.$ average absolute deviation), which can be explained by the contrasting way that $\Delta E(\zeta)$ is computed, i.e., using the difference between MP4 computations (ccCA-TZ) versus the difference between MP2 computations (ccCA-aTZ). This slim degradation in accuracy from using MP2 for the $\Delta E(\zeta)$ correction is tolerable since computations of MP4 cc-pVQZ or MP4 augcc-pVQZ energies would become the computational bottleneck of the ccCA methods and would limit the size of molecules that can be treated.

The mean absolute deviation of the ccCA-CBS- 1 and ccCA-CBS- 2 atomic IPs $\left(0.80\right.$ and $0.71 \mathrm{kcal} \mathrm{mol}^{-1}$, respectively) are comparable to the ccCA-TZ method and nearly half that of the $\mathrm{G} 3$ method $\left(1.24 \mathrm{kcal} \mathrm{mol}^{-1}\right)$. The largest ccCA-CBS-2 absolute deviation $\left(1.6 \mathrm{kcal} \mathrm{mol}^{-1}\right.$ for $\left.\mathrm{Na}^{+}\right)$, is less than half that of $\mathrm{G} 3\left(3.5 \mathrm{kcal} \mathrm{mol}^{-1}\right.$ for both $\mathrm{Mg}^{+}$and
$\mathrm{Be}^{+}$). It should be noted that the ccCA-CBS methods are faster and of equal or surpassed accuracy to the ccCA-TZ and ccCA-aTZ methods. Therefore, by removing basis set incompleteness effects and eliminating costly MP4 computations, the ccCA-CBS methods hold two distinct advantages over the ccCA-aTZ method.

Basis set deficiencies within the ccCA-DZ, ccCA-TZ, and ccCA-aTZ clearly arise when computing electron affinities; a traditionally more difficult atomic and molecular property to accurately describe. The ccCA-DZ method grossly overestimates electron affinities, with a maximum deviation of $-30.7 \mathrm{kcal} \mathrm{mol}^{-1}$ for $\mathrm{F}^{-}$and an average deviation of $-14.3 \mathrm{kcal} \mathrm{mol}^{-1}$. The average deviation is lessened but still unacceptable at the ccCA-TZ level, $-7.6 \mathrm{kcal} \mathrm{mol}^{-1}$. Our assessment is that the ccCA-DZ and ccCA-TZ $\Delta E(\zeta)$ additive correction for anions is overemphasized with the MP4 level of theory. Using the MP2 augmented CBS values as the reference energy seems to compensate for this problem. While it appears that the ccCA-aTZ method performs slightly worse than ccCA-TZ for atomic IPs, the ccCA-aTZ and ccCA-CBS methods perform splendidly for atomic EAs with 
TABLE III. Atomic ionization potentials and electron affinities (both in $\mathrm{kcal} \mathrm{mol}^{-1}$ ) with $\mathrm{G} n$ and ccCA methods.

\begin{tabular}{|c|c|c|c|c|c|c|c|c|c|}
\hline $\begin{array}{l}\text { Ionization } \\
\text { potential }\end{array}$ & Expt. ${ }^{a}$ & G1 & G2 & G3 & $\begin{array}{l}\text { ccCA- } \\
\text { DZ }\end{array}$ & $\begin{array}{l}\text { ccCA- } \\
\text { TZ }\end{array}$ & $\begin{array}{l}\text { ccCA- } \\
\text { aTZ }\end{array}$ & $\begin{array}{l}\text { ccCA- } \\
\text { CBS-1 }\end{array}$ & $\begin{array}{l}\text { ccCA- } \\
\text { CBS-2 }\end{array}$ \\
\hline $\mathrm{Li}$ & 124.3 & 123.2 & 124.5 & 124.5 & 123.5 & 123.9 & 123.9 & 123.9 & 123.9 \\
\hline $\mathrm{Be}$ & 214.9 & 217.7 & 218.1 & 218.4 & 214.0 & 215.2 & 215.7 & 216.4 & 216.3 \\
\hline B & 191.4 & 188.9 & 189.9 & 189.9 & 189.4 & 190.1 & 190.2 & 189.8 & 190.5 \\
\hline $\mathrm{C}$ & 259.7 & 257.6 & 258.5 & 258.6 & 259.1 & 258.9 & 258.8 & 259.0 & 259.2 \\
\hline $\mathrm{N}$ & 335.3 & 333.7 & 334.5 & 334.6 & 336.3 & 335.0 & 334.8 & 334.9 & 335.1 \\
\hline $\mathrm{O}$ & 313.9 & 312.3 & 312.4 & 312.7 & 314.0 & 313.8 & 312.1 & 313.2 & 313.1 \\
\hline $\mathrm{F}$ & 401.7 & 401.1 & 401.0 & 401.3 & 405.0 & 402.6 & 400.6 & 401.7 & 401.4 \\
\hline $\mathrm{Na}$ & 118.5 & 114.2 & 117.9 & 118.0 & 115.4 & 116.9 & 116.9 & 116.9 & 116.9 \\
\hline $\mathrm{Mg}$ & 176.3 & 177.0 & 179.5 & 179.8 & 174.8 & 176.3 & 176.7 & 177.0 & 177.0 \\
\hline $\mathrm{Al}$ & 138.0 & 136.7 & 137.4 & 137.5 & 137.3 & 137.2 & 137.4 & 137.8 & 137.8 \\
\hline $\mathrm{Si}$ & 187.9 & 186.7 & 187.4 & 187.5 & 188.2 & 187.7 & 187.7 & 187.9 & 188.0 \\
\hline $\mathrm{P}$ & 241.9 & 240.7 & 241.3 & 241.3 & 243.4 & 242.6 & 242.4 & 242.6 & 242.7 \\
\hline S & 238.9 & 235.5 & 236.8 & 237.1 & 237.0 & 237.5 & 236.5 & 237.6 & 237.6 \\
\hline $\mathrm{Cl}$ & 299.1 & 296.7 & 297.3 & 297.6 & 298.3 & 298.8 & 297.4 & 297.7 & 298.4 \\
\hline Mean Dev. IP & & 1.41 & 1.54 & 0.21 & 0.45 & 0.38 & 0.76 & 0.39 & 0.28 \\
\hline Mean Abs. Dev. & & 1.91 & 1.85 & 1.24 & 1.34 & 0.64 & 1.98 & 0.80 & 0.71 \\
\hline Standard Dev. & & 1.71 & 1.59 & 1.66 & 1.63 & 0.75 & 0.94 & 0.93 & 0.82 \\
\hline \multicolumn{10}{|l|}{ Electron affinity } \\
\hline $\mathrm{C}$ & 29.1 & 27.4 & 27.5 & 27.6 & 39.7 & 33.7 & 28.4 & 27.9 & 28.0 \\
\hline $\mathrm{O}$ & 33.7 & 32.5 & 30.8 & 31.1 & 56.5 & 45.1 & 33.7 & 33.8 & 33.5 \\
\hline $\mathrm{F}$ & 78.4 & 79.8 & 78.4 & 78.7 & 109.1 & 94.6 & 81.7 & 81.2 & 80.6 \\
\hline $\mathrm{Si}$ & 31.9 & 30.4 & 31.8 & 31.8 & 37.3 & 37.2 & 32.3 & 32.1 & 32.2 \\
\hline$P$ & 17.2 & 13.9 & 17.1 & 16.7 & 26.4 & 22.6 & 15.6 & 16.2 & 16.2 \\
\hline S & 47.9 & 45.1 & 47.6 & 47.9 & 58.8 & 52.7 & 46.9 & 48.1 & 47.7 \\
\hline $\mathrm{Cl}$ & 83.4 & 82.3 & 83.2 & 83.5 & 93.9 & 89.2 & 83.3 & 86.6 & 84.5 \\
\hline Mean Dev. EA & & 1.46 & 0.91 & 0.61 & -14.31 & -7.64 & -0.04 & -0.61 & -0.16 \\
\hline Mean Abs. Dev. & & 1.86 & 1.43 & 0.73 & 14.31 & 7.64 & 1.01 & 1.24 & 0.87 \\
\hline Standard Dev. & & 1.51 & 1.39 & 1.06 & 9.02 & 4.46 & 1.59 & 1.73 & 1.17 \\
\hline
\end{tabular}

${ }^{\mathrm{a}}$ See Refs. 2 and 4 for experimental references.

average absolute deviations of 1.01, 1.24, and $0.87 \mathrm{kcal} \mathrm{mol}^{-1}$ for ccCA-aTZ, ccCA-CBS-1, and ccCACBS-2, respectively. Though the basis set dependence of the ccCA methods has been shown here on only a small number of atomic properties, we find that molecular properties behave similarly. For example, the ccCA-TZ electron affinity of $\mathrm{CH}_{2}{ }^{-}$is predicted to be $19.5 \mathrm{kcal} \mathrm{mol}^{-1}$, compared to the experimental value of $15.0 \mathrm{kcal} \mathrm{mol}^{-1}$, while the ccCA-aTZ method predicts an EA of $14.4 \mathrm{kcal} \mathrm{mol}^{-1}$, and the ccCACBS-1 and ccCA-CBS-2 methods both predict an EA of $15.1 \mathrm{kcal} \mathrm{mol}^{-1}$.

\section{ASSESSMENT OF ccCA ON THE G2-1 TEST SET}

The G2-1 test set contains 125 atomic and molecular electronic energies, ${ }^{4}$ and from those energies, 55 enthalpies of formation at $298.15 \mathrm{~K}\left(\Delta H_{f}\right), 38$ adiabatic ionization potentials (IPs), 25 adiabatic electron affinities (EAs), and 8 adiabatic proton affinities (PAs) can be determined and compared to reliable experimental data.

\section{A. Performance of ccCA}

Due to the persistent discrepancies over the $\Delta H_{f}$ value of silicon gas, ${ }^{34,78-82}$ seven molecules from the G2-1 test set containing silicon atoms have been removed from our initial test set. Work is currently being carried out upon small silicon-containing compounds in order to assess and improve the errors of the ccCA-CBS methods, and more quantitatively address controversy in the gas phase enthalpy of formation of silicon. ${ }^{83}$

In Table IV, the G2-1 test set standard enthalpies of formation at $298.15 \mathrm{~K}$ predicted with G1, G2, G3B3, ccCAaTZ, ccCA-CBS-1, and ccCA-CBS-2 are compared. The mean deviations of the $48 \Delta H_{f}$ values computed with ccCAaTZ, ccCA-CBS-1, and ccCA-CBS-2 are $-0.93,1.02$, and $1.23 \mathrm{kcal} \mathrm{mol}^{-1}$, respectively. The mean absolute deviations for the three variants are $1.31,1.45$, and $1.33 \mathrm{kcal} \mathrm{mol}^{-1}$, all within the desired $\pm 2 \mathrm{kcal} \mathrm{mol}^{-1}$ range of near-chemical accuracy. Due to the nature of the HLC being optimized for a large test set that includes the G2-1 set, the G3B3 enthalpies of formation have a mean deviation of $0.03 \mathrm{kcal} \mathrm{mol}^{-1}$, with no discernable pattern to allow inferences as to why some enthalpies of formation are higher or lower than the experimental values. In contrast, the most problematic cases within the G2-1 test set for the ccCA-aTZ method are structurally similar; hydrocarbons and small systems with multiple bonds where the $\Delta H_{f}$ values are always overestimated compared to the experimental quantity. However, the ccCA-CBS methods perform much better for hydrocarbons and multiply bonded systems while most of the larger errors from the ccCA-CBS methods come from molecules containing second-row main 
TABLE IV. Computed enthalpies of formation (in $\mathrm{kcal} \mathrm{mol}^{-1}$ ) of G2-1 test set.

\begin{tabular}{|c|c|c|c|c|c|c|c|c|c|c|}
\hline \multirow[b]{2}{*}{ Species } & \multirow[b]{2}{*}{ Expt. $^{\mathrm{a}}$} & \multirow[b]{2}{*}{ G1 } & \multirow[b]{2}{*}{$\begin{array}{c}\text { G1 w/o } \\
\text { HLC }\end{array}$} & \multirow[b]{2}{*}{ G2 } & \multicolumn{2}{|c|}{$\Delta \mathrm{H}_{f}(298.15 \mathrm{~K})$} & \multirow[b]{2}{*}{$\begin{array}{l}\text { ccCA- } \\
\text { aTZ }\end{array}$} & \multirow[b]{2}{*}{$\begin{array}{l}\text { ccCA- } \\
\text { CBS-1 }\end{array}$} & \multirow[b]{2}{*}{$\begin{array}{l}\text { ccCA- } \\
\text { CBS-2 }\end{array}$} & \multirow[b]{2}{*}{$\begin{array}{c}+\mathrm{SO}+ \\
\mathrm{SR}^{\mathrm{b}}\end{array}$} \\
\hline & & & & & G3B3 & $\begin{array}{c}\text { G3B3 w/o } \\
\text { HLC }\end{array}$ & & & & \\
\hline \multicolumn{11}{|c|}{ G2-1 test set } \\
\hline $\mathrm{LiH}$ & 33.3 & 32.8 & 36.4 & 32.7 & 32.9 & 35.5 & 33.1 & 32.6 & 32.4 & 32.4 \\
\hline $\mathrm{BeH}$ & 81.7 & 83.6 & 83.6 & 83.2 & 82.3 & 83.1 & 82.0 & 81.8 & 81.7 & 81.7 \\
\hline $\mathrm{CH}$ & 142.4 & 142.4 & 146.0 & 141.9 & 141.0 & 144.4 & 142.8 & 142.6 & 142.2 & 142.3 \\
\hline $\mathrm{CH}_{2}\left({ }^{3} \mathrm{~B}_{1}\right)$ & 93.5 & 95.9 & 99.5 & 94.7 & 92.3 & 96.6 & 94.5 & 94.4 & 93.7 & 94.0 \\
\hline $\mathrm{CH}_{2}\left({ }^{1} \mathrm{~A}_{1}\right)$ & 102.5 & 102.1 & 109.4 & 101.4 & 101.8 & 106.2 & 103.1 & 102.5 & 101.9 & 102.1 \\
\hline $\mathrm{CH}_{3}$ & 35.1 & 36.8 & 44.0 & 35.1 & 34.3 & 40.2 & 36.3 & 35.7 & 34.8 & 35.1 \\
\hline $\mathrm{CH}_{4}$ & -17.9 & -16.4 & -5.5 & -18.6 & -17.9 & -9.9 & -16.1 & -16.9 & -18.0 & -17.7 \\
\hline $\mathrm{NH}$ & 85.2 & 87.0 & 90.6 & 86.3 & 84.1 & 88.8 & 86.6 & 86.0 & 85.8 & 85.9 \\
\hline $\mathrm{NH}_{2}$ & 44.5 & 46.7 & 53.9 & 45.0 & 44.3 & 50.2 & 45.7 & 44.5 & 44 & 44.2 \\
\hline $\mathrm{NH}_{3}$ & -11.0 & -8.5 & 2.4 & -10.8 & -10.3 & -3.0 & -9.3 & -11.2 & -11.8 & -11.5 \\
\hline $\mathrm{OH}$ & 8.9 & 10.2 & 13.8 & 9.1 & 8.4 & 11.3 & 9.2 & 8.3 & 8.2 & 8.6 \\
\hline $\mathrm{OH}_{2}$ & -57.8 & -55.7 & -48.5 & -58.1 & -57.6 & -53.3 & -57.6 & -59.4 & -59.5 & -59.0 \\
\hline $\mathrm{FH}$ & -65.1 & -64.4 & -60.8 & -66.2 & -65.2 & -63.8 & -65.8 & -66.6 & -66.7 & -66.1 \\
\hline $\mathrm{PH}_{2}$ & 33.1 & 34.0 & 41.2 & 32.9 & 32.4 & 38.4 & 33.0 & 31.6 & 31.3 & 31.7 \\
\hline $\mathrm{PH}_{3}$ & 1.3 & 3.4 & 14.3 & 2.0 & 2.8 & 10.1 & 2.4 & 0.3 & -0.1 & 0.5 \\
\hline $\mathrm{SH}_{2}$ & -4.9 & -3.0 & 4.2 & -4.8 & -4.5 & -0.2 & -5.8 & -7.2 & -7.5 & -6.4 \\
\hline $\mathrm{ClH}$ & -22.1 & -21.0 & -17.4 & -22.4 & -21.6 & -20.2 & -23.1 & -25.2 & -24.3 & -23.1 \\
\hline $\mathrm{Li}_{2}$ & 51.6 & 48.8 & 52.4 & 49.6 & 49.2 & 51.7 & 51.0 & 50.6 & 50.5 & 50.5 \\
\hline $\mathrm{LiF}$ & -80.1 & -85.2 & -81.6 & -81.4 & -80.4 & -79.0 & -81.4 & -83.4 & -82.8 & -81.7 \\
\hline $\mathrm{C}_{2} \mathrm{H}_{2}$ & 54.2 & 56.1 & 67.0 & 55.8 & 54.3 & 61.2 & 57.4 & 56.8 & 54.6 & 55.1 \\
\hline $\mathrm{C}_{2} \mathrm{H}_{4}$ & 12.5 & 14.4 & 28.8 & 12.8 & 12.3 & 21.7 & 15.4 & 13.4 & 12.0 & 12.6 \\
\hline $\mathrm{C}_{2} \mathrm{H}_{6}$ & -20.1 & -17.5 & 0.6 & -20.6 & -20.1 & -8.1 & -17.1 & -18.7 & -20.6 & -20.0 \\
\hline $\mathrm{CN}$ & 104.9 & 107.0 & 114.2 & 107.3 & 104.9 & 110.4 & 108.2 & 106.1 & 106.0 & 106.3 \\
\hline $\mathrm{HCN}$ & 31.5 & 30.9 & 41.7 & 31.2 & 30.6 & 37.5 & 33.9 & 31.4 & 30.8 & 31.2 \\
\hline $\mathrm{CO}$ & -26.4 & -27.9 & -20.6 & -28.2 & -27.0 & -23.1 & -25.0 & -27.8 & -27.7 & -27.2 \\
\hline $\mathrm{HCO}$ & 10.0 & 9.4 & 16.6 & 9.3 & 9.4 & 13.6 & 10.6 & 8.3 & 8.0 & 8.7 \\
\hline $\mathrm{H}_{2} \mathrm{CO}$ & -26.0 & -26.8 & -15.9 & -27.9 & -26.9 & -20.4 & -24.4 & -26.8 & -27.4 & -26.7 \\
\hline $\mathrm{H}_{3} \mathrm{COH}$ & -48.0 & -46.5 & -32.1 & -49.4 & -48.2 & -35.0 & -46.3 & -48.5 & -49.6 & -48.7 \\
\hline $\mathrm{N}_{2}$ & 0.0 & 0.3 & 11.1 & 1.3 & 0.6 & 7.5 & 3.0 & -0.5 & -0.5 & -0.3 \\
\hline $\mathrm{H}_{2} \mathrm{NNH}_{2}$ & 22.8 & 26.9 & 44.9 & 23.7 & 24.2 & 36.2 & 26.1 & 22.4 & 21.6 & 22.2 \\
\hline NO & 21.6 & 21.3 & 28.6 & 21.0 & 21.7 & 26.8 & 24.0 & 21.2 & 21.5 & 22.0 \\
\hline $\mathrm{O}_{2}$ & 0.0 & 2.6 & 6.2 & 2.4 & -0.1 & 3.3 & 1.8 & 0.1 & 0.2 & 0.9 \\
\hline $\mathrm{HOOH}$ & -32.5 & -30.7 & -19.9 & -32.3 & -31.9 & -25.8 & -31.0 & -33.2 & -33.7 & -32.8 \\
\hline $\mathrm{F}_{2}$ & 0.0 & -0.5 & 3.1 & 0.3 & 1.2 & 1.5 & 0.2 & 0.6 & -0.2 & 0.7 \\
\hline $\mathrm{CO}_{2}$ & -94.1 & -96.4 & -85.5 & -96.7 & -95.7 & -89.9 & -91.7 & -95.8 & -95.9 & -94.7 \\
\hline $\mathrm{Na}_{2}$ & 34.0 & 30.9 & 34.5 & 31.6 & 29.7 & 32.2 & 32.6 & 32.3 & 32.2 & 32.2 \\
\hline $\mathrm{P}_{2}$ & 34.3 & 34.4 & 45.2 & 35.7 & 34.9 & 41.8 & 37.4 & 33.1 & 33.7 & 34.2 \\
\hline $\mathrm{S}_{2}$ & 30.7 & 34.0 & 37.7 & 33.9 & 31.8 & 36.3 & 31.9 & 29.3 & 29.5 & 31.1 \\
\hline $\mathrm{Cl}_{2}$ & 0.0 & -0.8 & 2.8 & 1.4 & 1.9 & 2.2 & -0.5 & -4.3 & -2.6 & -0.6 \\
\hline $\mathrm{NaCl}$ & -43.6 & -45.5 & -41.9 & -44.8 & -44.6 & -43.2 & -43.7 & -47.0 & -45.4 & -44.1 \\
\hline $\mathrm{SC}$ & 66.9 & 65.1 & 72.3 & 65.9 & 65.9 & 69.9 & 67.4 & 64.4 & 64.8 & 65.8 \\
\hline SO & 1.2 & 4.4 & 8.1 & 3.8 & 1.5 & 4.9 & 1.9 & 0.0 & -0.3 & 1.0 \\
\hline $\mathrm{ClO}$ & 24.2 & 26.7 & 30.3 & 26.4 & 26.6 & 28.5 & 25.4 & 22.9 & 23.8 & 25.2 \\
\hline $\mathrm{FCl}$ & -13.2 & -14.1 & -10.5 & -14.0 & -12.0 & -11.7 & -14.4 & -16.8 & -15.7 & -14.2 \\
\hline $\mathrm{CH}_{3} \mathrm{Cl}$ & -19.6 & -19.1 & -8.3 & -20.5 & -19.1 & -13.0 & -19.2 & -21.8 & -21.9 & -20.5 \\
\hline $\mathrm{H}_{3} \mathrm{CSH}$ & -5.5 & -3.2 & 11.3 & -5.3 & -4.9 & 4.2 & -4.8 & -6.8 & -8.0 & -6.6 \\
\hline $\mathrm{HOCl}$ & -17.8 & -17.6 & -10.4 & -18.3 & -17.2 & -14.0 & -18.6 & -21.8 & -20.9 & -19.4 \\
\hline $\mathrm{SO}_{2}$ & -71.0 & -63.5 & -52.7 & -65.9 & -68.4 & -63.0 & -71.3 & -77.2 & -77.9 & -75.8 \\
\hline $\begin{array}{l}\text { Mean Dev. } \\
\text { Mean Abs. }\end{array}$ & & -0.80 & -8.33 & -0.13 & 0.03 & -4.88 & -0.93 & 1.02 & 1.23 & 0.51 \\
\hline Dev. & & 1.80 & 8.39 & 1.16 & 0.85 & 4.96 & 1.31 & 1.45 & 1.33 & 0.81 \\
\hline Std. Dev. & & 2.13 & 5.26 & 1.53 & 1.18 & 3.30 & 1.34 & 1.66 & 1.28 & 0.99 \\
\hline $\begin{array}{l}\text { Max. } \\
\text { Deviation }\end{array}$ & & $\mathrm{SO}_{2}(-7.5)$ & $\mathrm{H}_{2} \mathrm{NNH}_{2}(-22.1)$ & $\mathrm{SO}_{2}(-5.0)$ & $\begin{array}{l}\mathrm{Na}_{2} \\
(4.3)\end{array}$ & $\mathrm{H}_{2} \mathrm{NNH}_{2}(-13.4)$ & $\mathrm{CN} / \mathrm{H}_{2} \mathrm{NNH}_{2}(-3.3)$ & $\begin{array}{l}\mathrm{SO}_{2} \\
(6.2)\end{array}$ & $\begin{array}{l}\mathrm{SO}_{2} \\
(6.9)\end{array}$ & $\begin{array}{l}\mathrm{SO}_{2} \\
(4.8)\end{array}$ \\
\hline
\end{tabular}

${ }^{\mathrm{a}}$ See Refs. 2, 3, 6, 71, and 89 for experimental references.

${ }^{\mathrm{b}}$ Atomic spin-orbit (SO) corrections and scalar relativistic (SR) SDPT corrections from Refs. 84 and 86 are added to the ccCA-CBS-2 atomization energies. 
group atoms. For example, maximum deviation in ccCACBS theoretical $\Delta H_{f}$ values occurs with $\mathrm{SO}_{2}$, with the ccCACBS- 1 method in error by $+6.2 \mathrm{kcal} \mathrm{mol}^{-1}$ and the ccCACBS-2 method in error by $+6.9 \mathrm{kcal} \mathrm{mol}^{-1}$. Modifications of ccCA to improve the treatment of molecules with secondrow elements will be discussed in later sections.

The maximum deviation of the G3B3 method is $+4.3 \mathrm{kcal} \mathrm{mol}^{-1}$ with $\mathrm{Na}_{2}$, while the ccCA-aTZ maximum deviation is $-3.3 \mathrm{kcal} \mathrm{mol}^{-1}$ with $\mathrm{CN}$. The ccCA-aTZ, ccCACBS-1, and ccCA-CBS-2 methods, respectively, have 10, 11, and 10 species over the $\pm 2 \mathrm{kcal} \mathrm{mol}^{-1}$ range whereas only four systems are outside this error range using G3B3. Compared to using ccCA-aTZ, extrapolations to the one-particle CBS limit lower the total energy of molecules relative to the component atoms. This in turn raises computed atomization energies and proportionally lowers the computed value of $\Delta H_{f}$.

For the 38 adiabatic ionization potentials contained in the G2-1 test set (shown in Table V), ccCA-aTZ, ccCACBS-1, and ccCA-CBS-2 have mean absolute deviations of $0.63,0.91$, and $0.81 \mathrm{kcal} \mathrm{mol}^{-1}$, respectively. This compares well to G3B3, which has a mean absolute deviation of $0.89 \mathrm{kcal} \mathrm{mol}^{-1}$. The ccCA-CBS-2 has two IPs with deviations over $\pm 2 \mathrm{kcal} \mathrm{mol}^{-1}, \mathrm{CH}_{4}{ }^{+}$and $\mathrm{SC}^{+}$. Two atomic ionization potentials over the $\pm 2 \mathrm{kcal} \mathrm{mol}^{-1}$ error limit (Be and $\mathrm{Mg}$ ) occur using $\mathrm{G} 3 \mathrm{~B} 3$, while $\mathrm{CH}_{4}{ }^{+}$is the only molecular ionization potential in the G2-1 test set poorly described by G3B3.

The 25 computed adiabatic electron affinities (shown in Table VI) have ccCA-aTZ, ccCA-CBS-1, and ccCA-CBS-2 mean absolute deviations of $0.93,1.10$, and $1.02 \mathrm{kcal} \mathrm{mol}^{-1}$, respectively. Three of the ccCA-CBS-2 electron affinities exceed the $\pm 2 \mathrm{kcal} \mathrm{mol}^{-1}$ error range, compared to ten systems using G1, six using G2, three using G3, and four using G3B3. The eight proton affinities (shown in Table VII) have ccCA-aTZ, ccCA-CBS-1, and ccCA-CBS-2 mean absolute deviations of $1.48,1.49$, and $1.51 \mathrm{kcal} \mathrm{mol}^{-1}$, respectively. Because the protonated species have the same number of valence electrons as their neutral counterparts, their $\mathrm{G} n$ HLCs will be equivalent. Since there is no difference in the HLCs of the neutral and protonated species, removal of the HLC will not change the Gn PA values. Thus the $\mathrm{G} n$ methods slightly outperform ccCA for overall computation of proton affinities.

\section{B. Removal of the HLC}

In order to more directly compare the basis set difference between $\mathrm{G} n$ methods and ccCA, the high-level correction has been subtracted from the G1 and G3B3 atomic and molecular energies in order to assess the quality of the purely $a b$ initio portions of the $\mathrm{G} n$ methods. In Table IV, the deficiency of the G1 and G3 MP4/6-31G $(d)$ reference energy is obvious. Whereas G3B3 with the HLC only predicts four of the $48 \mathrm{G} 2-1 \Delta H_{f}$ values to have a deviation from experiment larger than $\pm 2 \mathrm{kcal} \mathrm{mol}^{-1}$, uncorrected G3B3 has 37 systems outside the range of near-chemical accuracy. The G1 method, with its supposedly more physically sound "isogyric" HLC, only has five G2-1 systems with absolute deviations within
$2 \mathrm{kcal} \mathrm{mol}^{-1}$ of the experimental values! Without the HLC, the mean absolute deviation for the 48 G2-1 enthalpies of formation (silicon-containing molecules excluded) with G3B3 is $4.96 \mathrm{kcal} \mathrm{mol}^{-1}$. The implementation of standard DFT with a large basis set $[\mathrm{B} 3 \mathrm{LYP} / 6-311+\mathrm{G}(3 d f, 2 p)]$ to compute G2-1 enthalpies of formation has a mean absolute deviation of only $2.36 \mathrm{kcal} \mathrm{mol}^{-1}$. $^{6}$ Almost all uncorrected G1 and G3B3 enthalpies of formation are severely overestimated, especially hydrocarbons and multiply bonded systems. The most problematic systems for uncorrected $\mathrm{G} n$ methods correlate well with the problematic systems using ccCA with an incomplete basis set treatment.

As a testament to the relative ease in computing ionization potentials rather than enthalpies of formation, the G3B3 method still performs reliably without the HLC. The mean absolute deviation of IPs for G3B3 without the HLC is $1.79 \mathrm{kcal} \mathrm{mol}^{-1}$, with 12 species outside the $2 \mathrm{kcal} \mathrm{mol}^{-1}$ range. Uncorrected electron affinities, however, are quite poor, with an average deviation of $3.15 \mathrm{kcal} \mathrm{mol}^{-1}$ and all but five systems outside the $2 \mathrm{kcal} \mathrm{mol}^{-1}$ accuracy range. The noncorrected G3B3 always overestimates both IPs and EAs, except for two cases, $\mathrm{CH}_{4}{ }^{+}$and $\mathrm{SC}^{+}$.

\section{Atomic spin-orbit corrections}

Enthalpies of formation predicted by the ccCA-CBS- $n$ methods are predominantly overestimated, whereas the G3 and G3B3 enthalpies of formation are more evenly distributed around the experimental result. For G3 methods, this is because effects such as atomic spin-orbit splitting are folded into the total energies, and hence the HLC parametrization. Removal of atomic spin-orbit effects from the G3 methods will lower atomization energies. This trend, pictorially represented in Fig. 1, is the basis for our predilection towards the ccCA-CBS- 2 method. Of the 48 nonsilicon-containing enthalpies of formation within the G2-1 test set, all have a negative deviation except $\mathrm{Na}_{2}$ when employing the G3B3 method without a high-level correction. Removal of atomic spin-orbit corrections will shift calculated $\Delta H_{f}$ deviations even further in the negative direction. The ccCA-aTZ method predicts 35 values of $\Delta H_{f}$ with a negative deviation, while conversely the ccCA-CBS-1 method predicts 13 systems with negative deviation in $\Delta H_{f}$, and the ccCA-CBS-2 method predicts only 4 systems with a negative deviation. When atomic spin-orbit splitting and relativistic effects are computed in conjunction with the ccCA-CBS- $n$ methods, these combined corrections will generally lower atomization energies and proportionally raise the computed $\Delta H_{f}$, therefore increasing the overall accuracy in computing $\Delta H_{f}$. Using the same spin-orbit corrections as those in the original G3 method, ${ }^{5}$ the average $\Delta H_{f}$ deviation of the ccCA-aTZ method is more negative $\left(-0.95\right.$ to $\left.-1.30 \mathrm{kcal} \mathrm{mol}^{-1}\right)$ while the ccCA-CBS-1 average deviation improves, changing from +1.03 to $+0.65 \mathrm{kcal} \mathrm{mol}^{-1}$. Mean absolute deviations of the various composite methods are shown in Fig. 2. The ccCACBS-2 average deviation in $\Delta H_{f}$ improves from +1.24 to $+0.55 \mathrm{kcal} \mathrm{mol}^{-1}$. Due to the shift in atomization energies, the mean absolute deviation of the ccCA-aTZ method for the entire data set worsens from 1.29 to $1.46 \mathrm{kcal} \mathrm{mol}^{-1}$, while 
TABLE V. Computed ionization potentials (in $\mathrm{kcal} \mathrm{mol}^{-1}$ ) of G2-1 test set.

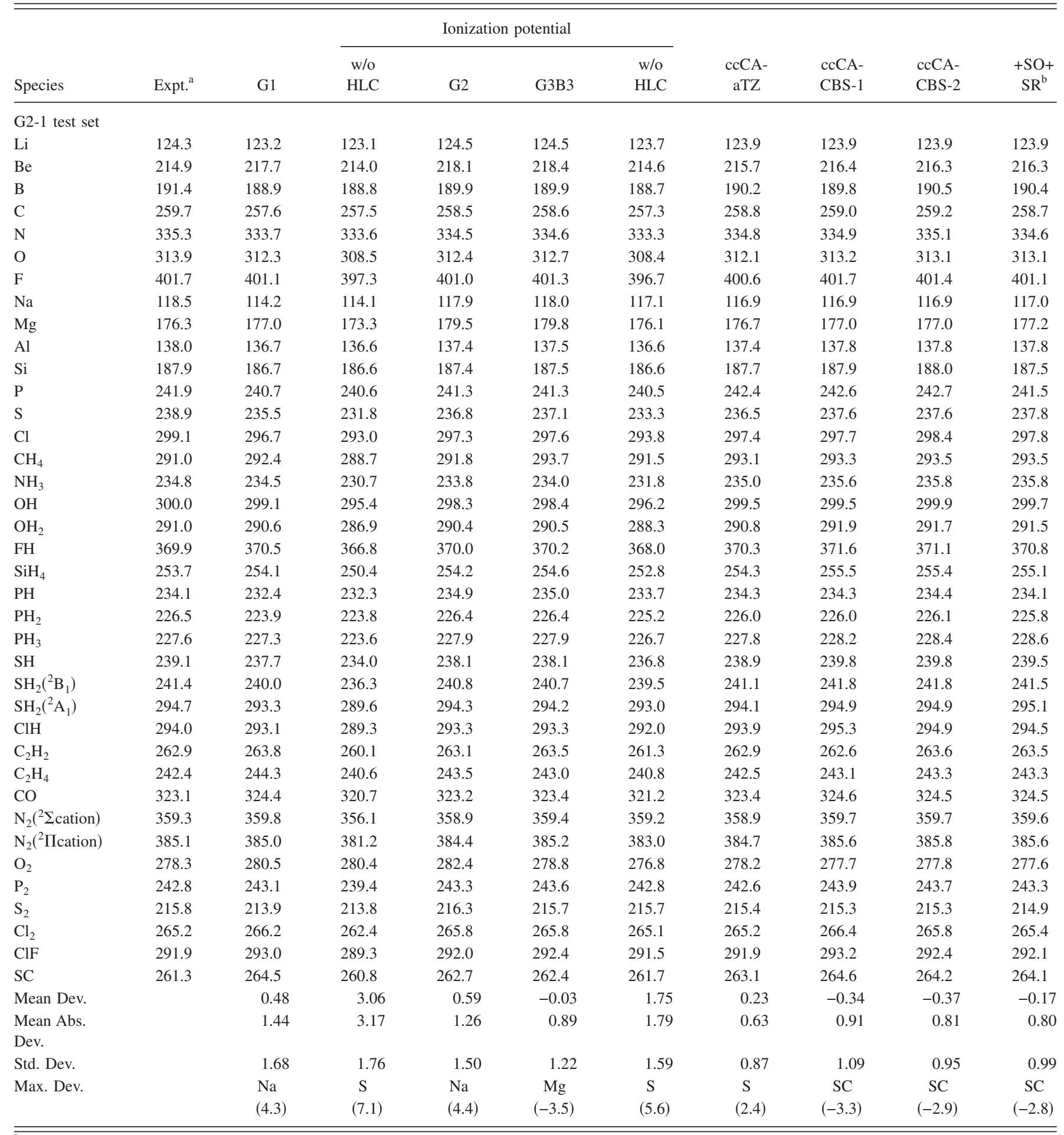

${ }^{\mathrm{a}}$ See Refs. 2 and 4 for experimental references.

${ }^{\mathrm{b}}$ Atomic spin-orbit (SO) corrections and scalar relativistic (SR) SDPT corrections from Refs. 84 and 86 are added to the ccCA-CBS-2 total energies.

ccCA-CBS-1 improves from 1.47 to $1.17 \mathrm{kcal} \mathrm{mol}^{-1}$, and ccCA-CBS-2 improves from 1.34 to $0.84 \mathrm{kcal} \mathrm{mol}^{-1}$. The inclusion of spin-orbit effects will also affect the computed atomic IPs and EAs, but has little effect on the overall deviation of these two quantities.

\section{Scalar relativistic effects}

Relativistic effects are obviously significant when comparing atoms or molecules containing elements including and beyond the $3 d$ transition metals. However, in investigations of relativistic effects on the molecular energies of the G3/99 test set, Kedziora and co-workers ${ }^{84-86}$ have found that scalar relativistic effects can have a contribution to atomization energies that is the same order of magnitude as atomic spinorbit splitting effects. To generate an approximation of scalar relativistic corrections, we have shifted G2-1 atomization energies using the G3large stationary direct perturbation theory $^{87}$ (SDPT) results obtained by Kedziora and 
TABLE VI. Computed electron affinities (in $\mathrm{kcal} \mathrm{mol}^{-1}$ ) of G2-1 test set.

\begin{tabular}{|c|c|c|c|c|c|c|c|c|c|c|}
\hline \multirow[b]{2}{*}{ Species } & \multirow[b]{2}{*}{ Expt. $^{\mathrm{a}}$} & \multirow[b]{2}{*}{ G1 } & \multirow[b]{2}{*}{$\begin{array}{l}\text { w/o } \\
\text { HLC }\end{array}$} & \multirow[b]{2}{*}{ G2 } & \multirow[b]{2}{*}{ G3B3 } & \multicolumn{5}{|c|}{ Electron affinity } \\
\hline & & & & & & $\begin{array}{l}\text { w/o } \\
\text { HLC }\end{array}$ & $\begin{array}{l}\text { ccCA- } \\
\text { aTZ }\end{array}$ & $\begin{array}{c}\text { ccCA-CBS- } \\
1\end{array}$ & $\begin{array}{l}\text { ccCA- } \\
\text { CBS-2 }\end{array}$ & $\begin{array}{l}+\mathrm{SO}^{\mathrm{b}} \\
\mathrm{SR}^{\mathrm{b}}\end{array}$ \\
\hline \multicolumn{11}{|l|}{ G2-1 test set } \\
\hline $\mathrm{C}$ & 29.1 & 27.4 & 27.2 & 27.5 & 27.6 & 27.2 & 28.4 & 27.9 & 28.0 & 27.9 \\
\hline $\mathrm{O}$ & 33.7 & 32.5 & 28.8 & 30.8 & 31.1 & 28.5 & 33.7 & 33.8 & 33.5 & 33.3 \\
\hline $\mathrm{F}$ & 78.4 & 79.8 & 76.1 & 78.4 & 78.7 & 76.4 & 81.7 & 81.2 & 80.6 & 80.3 \\
\hline $\mathrm{Si}$ & 31.9 & 30.4 & 30.3 & 31.8 & 31.8 & 28.3 & 32.3 & 32.1 & 32.2 & 32.0 \\
\hline$P$ & 17.2 & 13.9 & 10.2 & 17.1 & 16.7 & 13.7 & 15.6 & 16.2 & 16.2 & 15.9 \\
\hline S & 47.9 & 45.1 & 41.4 & 47.6 & 47.9 & 44.2 & 46.9 & 48.1 & 47.7 & 47.4 \\
\hline $\mathrm{Cl}$ & 83.4 & 82.3 & 78.6 & 83.2 & 83.5 & 79.8 & 83.3 & 86.6 & 84.5 & 84.1 \\
\hline $\mathrm{CH}$ & 28.6 & 25.5 & 25.4 & 27.2 & 27.1 & 25.0 & 27.2 & 27.2 & 27.2 & 27.1 \\
\hline $\mathrm{CH}_{2}$ & 15.0 & 14.9 & 11.2 & 13.4 & 13.0 & 10.8 & 14.4 & 15.1 & 15.1 & 15.1 \\
\hline $\mathrm{CH}_{3}$ & 1.8 & 0.3 & -3.5 & -0.9 & -1.2 & -3.4 & 0.0 & 1.0 & 1.0 & 1.0 \\
\hline NH & 8.8 & 6.5 & 2.8 & 4.5 & 4.4 & 2.4 & 6.5 & 6.5 & 6.7 & 6.6 \\
\hline $\mathrm{NH}_{2}$ & 17.8 & 17.1 & 13.4 & 16.1 & 15.9 & 13.7 & 18.6 & 19.8 & 19.7 & 19.6 \\
\hline $\mathrm{OH}$ & 42.2 & 42.6 & 38.8 & 41.0 & 41.0 & 38.8 & 45.1 & 45.2 & 45.0 & 44.8 \\
\hline $\mathrm{SiH}$ & 29.4 & 26.4 & 26.3 & 29.3 & 29.4 & 27.4 & 28.7 & 28.6 & 28.7 & 28.5 \\
\hline $\mathrm{SiH}_{2}$ & 25.9 & 22.1 & 22.1 & 24.8 & 24.8 & 22.8 & 24.5 & 24.5 & 24.6 & 24.4 \\
\hline $\mathrm{SiH}_{3}$ & 32.5 & 32.1 & 28.4 & 32.9 & 32.8 & 30.6 & 32.5 & 32.9 & 33.0 & 33.1 \\
\hline PH & 23.8 & 21.0 & 17.4 & 22.6 & 22.6 & 20.4 & 22.8 & 22.2 & 22.2 & 22.0 \\
\hline $\mathrm{PH}_{2}$ & 29.3 & 27.6 & 23.9 & 29.3 & 29.2 & 27.0 & 29.2 & 29.9 & 29.9 & 29.7 \\
\hline $\mathrm{SH}$ & 54.4 & 51.6 & 47.8 & 53.5 & 53.5 & 51.3 & 53.6 & 54.8 & 54.5 & 54.2 \\
\hline $\mathrm{O}_{2}$ & 10.1 & 10.9 & 7.2 & 9.2 & 8.6 & 6.3 & 10.2 & 9.2 & 9.3 & 9.1 \\
\hline NO & 0.5 & -1.4 & -1.5 & -0.1 & 0.0 & -2.0 & 0.5 & -0.2 & -0.2 & -0.4 \\
\hline $\mathrm{CN}$ & 89.0 & 93.0 & 89.3 & 90.6 & 89.4 & 87.2 & 89.5 & 90.6 & 90.6 & 90.6 \\
\hline $\mathrm{PO}$ & 25.1 & 25.4 & 25.2 & 22.6 & 25.5 & 23.4 & 25.2 & 24.7 & 24.6 & 24.3 \\
\hline $\mathrm{S}_{2}$ & 38.3 & 37.9 & 34.1 & 38.5 & 38.5 & 36.3 & 37.8 & 38.2 & 38.1 & 37.8 \\
\hline $\mathrm{Cl}_{2}$ & 55.1 & 52.2 & 52.1 & 56.9 & 56.3 & 54.3 & 54.0 & 54.8 & 53.3 & 53.2 \\
\hline Mean Dev. & & 1.28 & 3.85 & 0.80 & 0.84 & 3.15 & 0.28 & -0.07 & 0.13 & 0.30 \\
\hline Mean Abs. Dev. & & 1.84 & 3.88 & 1.32 & 1.08 & 3.15 & 0.93 & 1.10 & 1.02 & 1.09 \\
\hline Std. Dev. & & 1.78 & 1.97 & 1.40 & 1.28 & 1.29 & 1.27 & 1.46 & 1.28 & 1.27 \\
\hline Max. Dev. & $(-4.0)$ & $\begin{array}{l}\mathrm{CN} \\
(7.0)\end{array}$ & $\begin{array}{c}\mathrm{P} \\
(3.1)\end{array}$ & $\begin{array}{l}\mathrm{SiH}_{2} \\
(4.4)\end{array}$ & $\begin{array}{l}\mathrm{NH} \\
(6.4)\end{array}$ & $\begin{array}{c}\mathrm{NH} \\
(-3.3)\end{array}$ & $\begin{array}{c}F \\
(-3.2)\end{array}$ & $\begin{array}{c}\mathrm{Cl} \\
(-2.8)\end{array}$ & $\begin{array}{c}\mathrm{OH} \\
(-2.6)\end{array}$ & $\mathrm{OH}$ \\
\hline
\end{tabular}

${ }^{\mathrm{a}}$ See Refs. 2 and 4 for experiemntal references.

${ }^{\mathrm{b}}$ Atomic spin-orbit (SO) corrections and scalar relativistic (SR) SDPT corrections from Refs. 84 and 86 are added to the ccCA-CBS-2 total energies.

co-workers. ${ }^{84,86}$ Of all 55 enthalpies of formation in the G2-1 test set, only the $\mathrm{Na}_{2}$ system shows an increase in atomization energy when scalar relativistic effects are included. Thus, implemented scalar relativistic corrections will increase the average deviation in $\Delta H_{f}$ for G3 methods as well as the ccCA-aTZ method. Curtiss and co-workers have noted $^{88}$ that scalar relativistic effects do not have a significant effect on the accuracy of G3 methods once the HLC has been reparametrized. Though the G3 relativistic HLC parameters are not revealed in Ref. 88, it is likely that if the bias of

TABLE VII. Computed proton affinities (in $\mathrm{kcal} \mathrm{mol}^{-1}$ ) of G2-1 test set.

\begin{tabular}{|c|c|c|c|c|c|c|c|c|}
\hline \multirow[b]{2}{*}{ Species } & \multirow[b]{2}{*}{ Expt. $^{\mathrm{a}}$} & \multicolumn{4}{|c|}{ Proton affinity } & \multirow[b]{2}{*}{ ccCA-CBS-1 } & \multirow[b]{2}{*}{ ccCA-CBS-2 } & \multirow[b]{2}{*}{$+\mathrm{SR}^{\mathrm{b}}$} \\
\hline & & G1 & G2 & G3B3 & ccCA-aTZ & & & \\
\hline \multicolumn{9}{|c|}{ G2-1 test set } \\
\hline $\mathrm{NH}_{3}$ & 202.5 & 202.7 & 203.1 & 203.0 & 202.1 & 202.0 & 202.1 & 202.1 \\
\hline $\mathrm{OH}_{2}$ & 165.1 & 163.4 & 163.4 & 163.3 & 163.0 & 162.8 & 163.0 & 162.9 \\
\hline $\mathrm{C}_{2} \mathrm{H}_{2}$ & 152.3 & 152.5 & 152.8 & 152.3 & 148.7 & 149.3 & 148.5 & 148.5 \\
\hline $\mathrm{SiH}_{4}$ & 154.0 & 152.9 & 152.3 & 151.8 & 152.1 & 151.6 & 151.8 & 151.9 \\
\hline $\mathrm{PH}_{3}$ & 187.1 & 186.1 & 185.3 & 185.4 & 185.7 & 186.2 & 186.1 & 185.8 \\
\hline $\mathrm{SH}_{2}$ & 168.8 & 167.8 & 167.0 & 167.3 & 167.7 & 167.6 & 167.8 & 167.8 \\
\hline $\mathrm{ClH}$ & 133.6 & 132.3 & 132.6 & 132.7 & 133.3 & 132.7 & 133.2 & 133.2 \\
\hline $\mathrm{H}_{2}$ & 100.8 & & 99.3 & 99.6 & 99.7 & 99.6 & 99.6 & 99.6 \\
\hline
\end{tabular}

${ }^{\mathrm{a}}$ See Refs. 2 and 4 for experimental references.

${ }^{\mathrm{b}}$ Scalar relativistic (SR) SDPT corrections from Refs. 84 and 86 are added to the ccCA-CBS-2 total energies. 


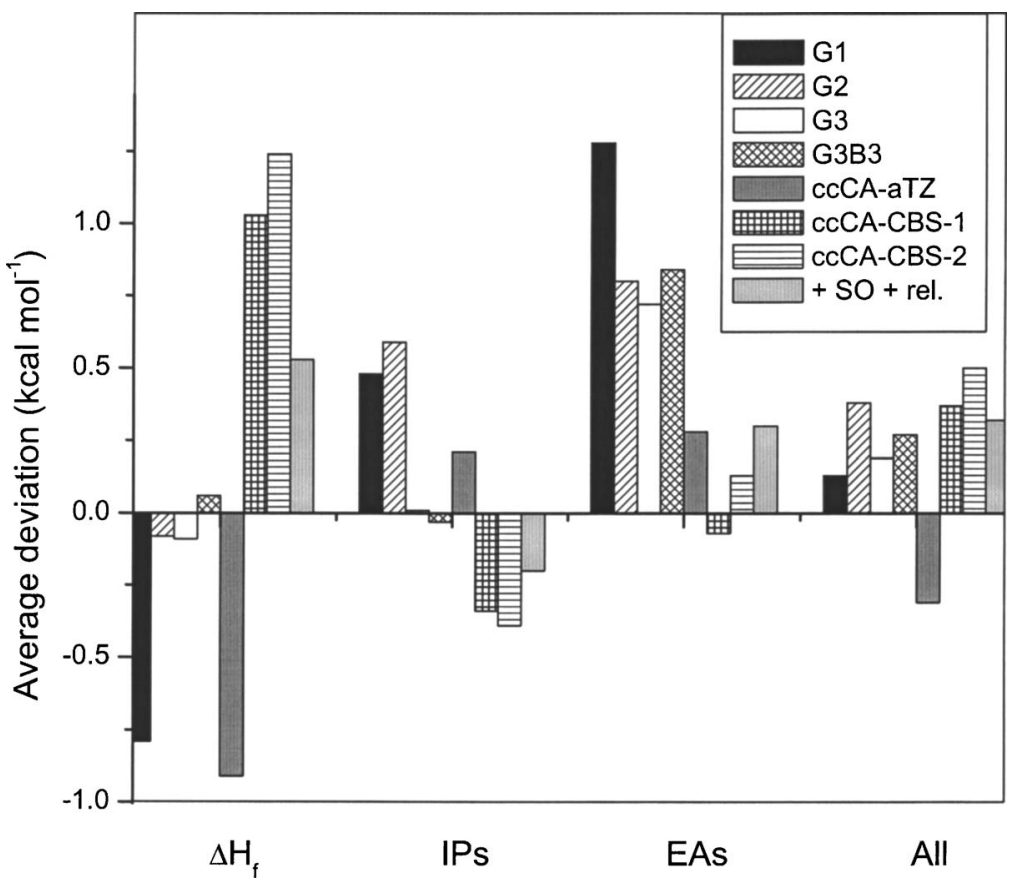

FIG. 1. Signed errors (in $\mathrm{kcal} \mathrm{mol}^{-1}$ ) for the various methods applied to experimentally known properties of the G2-1 test set.

the relativistic correction on enthalpies of formation uniformly increases the deviation from experiment in the same direction as basis set and spin-orbit effects, then the molecular HLC must increase in magnitude in order to account for more physical phenomena.

With the G3large SDPT relativistic correction, the mean absolute deviations in the 48 theoretical $\Delta H_{f}$ values with the ccCA-CBS-1 and ccCA-CBS-2 methods are 1.02 and $0.81 \mathrm{kcal} \mathrm{mol}^{-1}$. This ccCA-CBS-2 mean absolute deviation for the G2-1 test set, obtained with no empirical or optimized parameters, is $0.04 \mathrm{kcal} \mathrm{mol}^{-1}$ lower than that of G3B3. The scalar relativistic correction has a slight worsening effect on the accuracy of IPs, EAs, and PAs, but the change in the mean absolute deviations of all three of these quantities is less than $0.1 \mathrm{kcal} \mathrm{mol}^{-1}$. The average absolute deviation of 38 IPs is $0.81 \mathrm{kcal} \mathrm{mol}^{-1}, 1.09 \mathrm{kcal} \mathrm{mol}^{-1}$ for the $25 \mathrm{EAs}$, and $1.55 \mathrm{kcal} \mathrm{mol}^{-1}$ for the 8 PAs. Concerning these four major benchmarking experimental properties, ccCA-CBS-2 outperforms or matches the accuracy of the G3B3 method for all but the computation of proton affinities. Future studies will explore possible basis set, method, and geometry dependence of the relativistic corrections. Finally, after the scalar relativistic and spin-orbit effects are added to the 119 experimental values, the ccCA-CBS-2 method has an average deviation of $0.30 \mathrm{kcal} \mathrm{mol}^{-1}$ and an average absolute deviation of $0.92 \mathrm{kcal} \mathrm{mol}^{-1}$. For this data set, the average absolute deviation of the ccCA-CBS-2 method almost exactly matches that of G3B3 $\left(0.93 \mathrm{kcal} \mathrm{mol}^{-1}\right)$. The ccCA-CBS-2 method achieves this accuracy without employing empirical corrections, nor involving expensive coupled cluster computations.

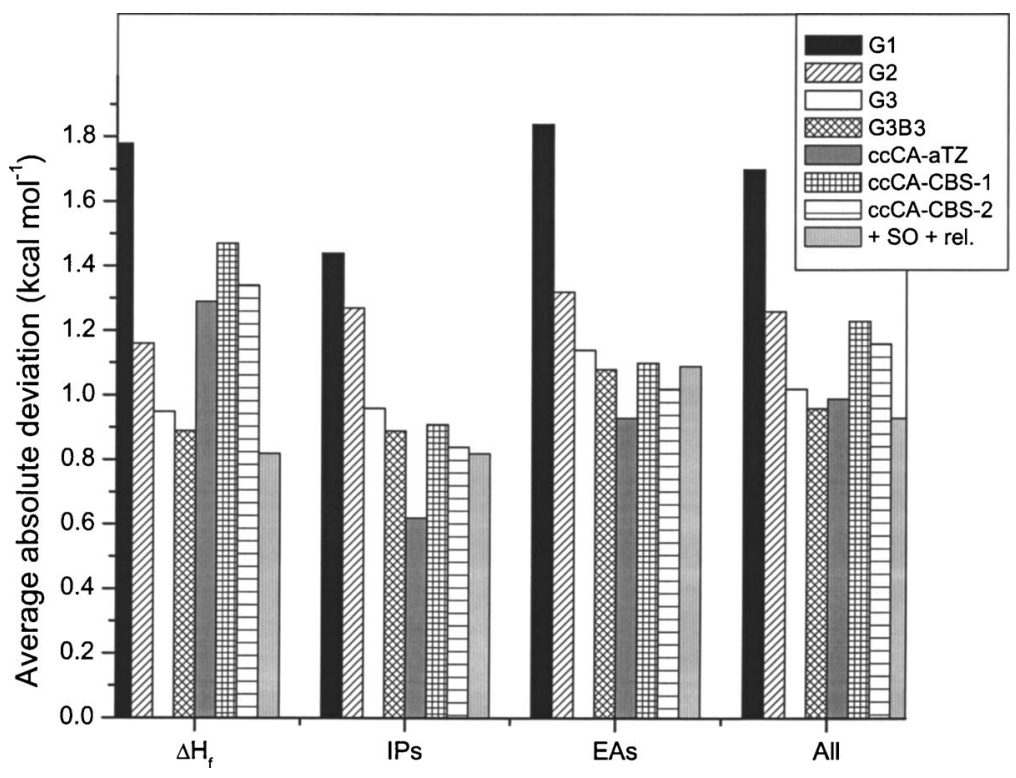

FIG. 2. Average absolute deviations (in $\mathrm{kcal} \mathrm{mol}^{-1}$ ) for the various methods applied to experimentally known properties of the G2-1 test set. 
TABLE VIII. Computed enthalpies of formation (in kcal mol${ }^{-1}$ ) of larger training set.

\begin{tabular}{|c|c|c|c|c|c|c|c|c|c|}
\hline \multirow[b]{2}{*}{ Species } & \multirow[b]{2}{*}{ Expt. $^{\mathrm{a}}$} & \multirow[b]{2}{*}{ G2 } & \multirow[b]{2}{*}{ G3B3 } & \multirow[b]{2}{*}{$\begin{array}{l}\text { ccCA- } \\
\text { aTZ }\end{array}$} & \multicolumn{2}{|c|}{$\Delta \mathrm{H}_{f}(298 \mathrm{~K})$} & \multirow[b]{2}{*}{$\begin{array}{l}\mathrm{ccCA}-\mathrm{aTZ} \\
+\mathrm{SO}+\mathrm{SR}^{\mathrm{b}}\end{array}$} & \multirow[b]{2}{*}{$\begin{array}{c}\text { ccCA-CBS-1 } \\
+\mathrm{SO}+\mathrm{SR}^{\mathrm{b}}\end{array}$} & \multirow[b]{2}{*}{$\begin{array}{c}\mathrm{ccCA}-\mathrm{CBS}-2 \\
+\mathrm{SO}+\mathrm{SR}^{\mathrm{b}}\end{array}$} \\
\hline & & & & & $\begin{array}{l}\text { ccCA- } \\
\text { CBS-1 }\end{array}$ & $\begin{array}{l}\text { ccCA- } \\
\text { CBS-2 }\end{array}$ & & & \\
\hline $\mathrm{BCl}_{3}$ & -96.3 & -98.3 & -95.1 & -96.4 & -104.3 & -100.9 & -92.9 & -100.8 & -97.4 \\
\hline $\mathrm{F}_{2} \mathrm{O}$ & 5.9 & 5.3 & 6.7 & 6.3 & 6.2 & 5.2 & 7.6 & 7.5 & 6.5 \\
\hline $\mathrm{CF}_{4}$ & -223.0 & -228.6 & -223.1 & -223.1 & -224.9 & -226.6 & -220.3 & -222.1 & -223.8 \\
\hline $\mathrm{C}_{2} \mathrm{~F}_{4}$ & -157.4 & -165.6 & -161.7 & -159.0 & -162.3 & -164.0 & -155.9 & -159.2 & -160.9 \\
\hline $\mathrm{CCl}_{4}$ & -22.9 & -25.7 & -23.0 & -24.5 & -33.7 & -29.5 & -20.1 & -29.4 & -25.1 \\
\hline $\mathrm{AlCl}_{3}$ & -139.7 & -142.5 & -141.6 & -142.2 & -151.5 & -147.9 & -137.8 & -147.1 & -143.5 \\
\hline $\mathrm{PF}_{3}$ & -229.1 & -223.7 & -223.3 & -227.6 & -231.5 & -232.7 & -225.2 & -229.1 & -230.3 \\
\hline $\mathrm{SF}_{6}$ & -291.7 & & -291.2 & -294.0 & -300.5 & -302.4 & -287.1 & -293.6 & -295.5 \\
\hline \multicolumn{10}{|l|}{ Linear alkanes } \\
\hline $\mathrm{C}_{3} \mathrm{H}_{8}$ (propane) & -25.0 & -25.4 & -25.0 & -20.8 & -23.2 & -25.8 & -19.9 & -22.3 & -24.9 \\
\hline $\mathrm{C}_{4} \mathrm{H}_{10}$ (n-butane) & -30.0 & -30.4 & -30.0 & -25.0 & -28.3 & -31.6 & -23.8 & -27.1 & -30.4 \\
\hline $\mathrm{C}_{5} \mathrm{H}_{12}$ (n-pentane) & -35.1 & & -34.9 & -29.6 & -32.7 & -36.7 & -28.0 & -31.1 & -35.1 \\
\hline $\mathrm{C}_{6} \mathrm{H}_{14}(n$-hexane $)$ & -39.9 & & -40.0 & -32.0 & -36.9 & -41.6 & -30.1 & -35.0 & -39.7 \\
\hline $\mathrm{C}_{8} \mathrm{H}_{18}$ (n-octane) & -49.9 & & -50.0 & & -45.9 & -52.1 & & $-43.4^{\mathrm{c}}$ & $-49.6^{c}$ \\
\hline \multicolumn{10}{|l|}{ Other hydrocarbons } \\
\hline $\mathrm{C}_{3} \mathrm{H}_{4}$ (cyclopropene) & 68.2 & 69.1 & 68.2 & 72.2 & 69.6 & 67.5 & 73.1 & 70.5 & 68.4 \\
\hline $\mathrm{C}_{4} \mathrm{H}_{10}$ (isobutane) & -32.1 & -32.4 & -31.9 & -26.8 & -30.1 & -33.4 & -25.6 & -28.9 & -32.2 \\
\hline $\mathrm{C}_{6} \mathrm{H}_{6}$ (benzene) & 19.7 & 23.7 & 20.3 & 28.5 & 22.6 & 19.5 & 30.7 & 24.3 & 21.2 \\
\hline $\mathrm{C}_{10} \mathrm{H}_{8}$ (naphthalene) & 36.1 & & 34.6 & 49.6 & 40.4 & 35.5 & 52.6 & 43.4 & 38.5 \\
\hline \multicolumn{10}{|l|}{ Substituted hydrocarbons } \\
\hline $\mathrm{CH}_{3} \mathrm{NH}_{2}$ (methylamine) & -5.5 & -5.5 & -4.6 & -2.1 & -4.8 & -6.1 & -1.5 & -4.2 & -5.5 \\
\hline $\mathrm{HCOOCH}_{3}$ (methyl formate) & -85.0 & -88.8 & -86.9 & -82.1 & -86.2 & -87.8 & -80.8 & -84.9 & -86.5 \\
\hline $\mathrm{C}_{2} \mathrm{H}_{5} \mathrm{OCH}_{3}$ (methyl ethyl ether) & -51.7 & -54.0 & -52.7 & -47.5 & -50.8 & -53.7 & -46.0 & -49.3 & -52.3 \\
\hline $\mathrm{C}_{6} \mathrm{H}_{5} \mathrm{Cl}$ (chlorobenzene) & 12.4 & & 12.1 & 19.7 & 11.8 & 10.0 & 21.5 & 13.6 & 12.8 \\
\hline $\mathrm{C}_{2} \mathrm{H}_{6} \mathrm{O}$ (ethanol) & -56.2 & -57.2 & -56.3 & -53.0 & -56.0 & -57.8 & -51.8 & -54.8 & -56.6 \\
\hline \multicolumn{10}{|l|}{ Organic radicals } \\
\hline $\mathrm{CH}_{3} \mathrm{O}$ & 5.0 & 4.8 & 4.4 & 6.9 & 5.5 & 4.5 & 7.6 & 6.2 & 5.2 \\
\hline $\mathrm{H}_{2} \mathrm{COH}$ & -4.1 & -3.8 & -4.1 & -2.2 & -4.2 & -5.1 & -1.4 & -3.4 & -4.3 \\
\hline $\mathrm{CH}_{3} \mathrm{CO}$ & -2.5 & -2.8 & -2.7 & 1.0 & -1.9 & -3.1 & 2.0 & -0.9 & -2.1 \\
\hline $\mathrm{CH}_{3} \mathrm{CH}_{2} \mathrm{O}$ & -3.3 & -2.3 & -2.4 & 0.4 & -1.8 & -3.6 & 1.7 & -0.5 & -2.3 \\
\hline \multicolumn{10}{|l|}{ Inorganic hydrides } \\
\hline $\mathrm{H}_{2}$ & 0.0 & -1.1 & -0.4 & 0.1 & -0.1 & -0.4 & 0.1 & -0.1 & -0.4 \\
\hline HS & 33.7 & 34.4 & 33.7 & 33.8 & 33.1 & 33.0 & 34.6 & 33.9 & 33.8 \\
\hline
\end{tabular}

${ }^{\mathrm{a}}$ See Refs. 2, 3, 6, 71, and 89 for experimental references.

${ }^{\mathrm{b}}$ Atomic spin-orbit (SO) corrections and scalar relativistic (SR) SDPT corrections from Refs. 84 and 86 are added to ccCA-CBS-2 total energies.

${ }^{\mathrm{c}} \mathrm{Scalar}$ relativistic correction is taken from SDPT atomization energy correction in Ref. 84 using the 6-31G $(d)$ basis set.

\section{SPECIFIC TEST CASES}

The ccCA methods were tested on a small subset of the G2-2 and G3/99 molecules as well as $\mathrm{C}\left(\mathrm{NO}_{2}\right)_{3}$ and adamantane from the set of 600 molecules compiled by Cioslowski et $a .^{41}$ Benchmarking of the ccCA methods on larger training sets will be published in due course, but we believe that merits and deficiencies of the ccCA methods will be evident in the following discussion.

\section{A. Some G2-2 / G3/99 test cases}

Table VIII displays the ccCA $\Delta H_{f}$ results for a selection of 28 systems from the G2-2 and G3/99 test sets. We have attempted to include some common organic and nonhydrocarbon species that perform with varying degrees of success when using G3 and G3B3. Some of the radical organic mol- ecules from the G2-2 test set whose enthalpies of formation have recently been revised by Ruscic et al. ${ }^{89}$ are also included.

When employing the ccCA methods with larger test cases, similar patterns as those discussed for the G2-1 test set emerge. The mean deviation for all types of molecules with the G3B3 method is very close to zero, an indicator of the success of the HLC in accounting for the variety of basis set and correlation deficiencies in the Gaussian- $n$ composite methods. The ccCA-aTZ method performs best with nonhydrocarbon species, resulting in a mean deviation of $+0.78 \mathrm{kcal} \mathrm{mol}^{-1}$ for the 8 systems studied, and performs poorly with both hydrocarbons and substituted hydrocarbons resulting in a mean deviation of $-5.54 \mathrm{kcal} \mathrm{mol}^{-1}$ for the 14 organic systems. As with the systems from the G2-1 training set, ccCA-CBS methods predict $\Delta H_{f}$ values significantly 
higher than the experimental values for nonhydrocarbons. The ccCA-CBS-1 method generally overestimates $\Delta H_{f}$ values for organic species, while the ccCA-CBS-2 method underestimates $\Delta H_{f}$. Once spin-orbit splitting and scalar relativistic effects are included, enthalpies of formation increase thereby improving the accuracy of the ccCA-CBS-2 method for all 28 species. The mean deviation of the ccCA-CBS-2 method is $0.51 \mathrm{kcal} \mathrm{mol}^{-1}$ while the absolute mean deviation is $1.16 \mathrm{kcal} \mathrm{mol}^{-1}$, comparable to the absolute mean deviation of the G3B3 method (0.99 kcal mol$\left.{ }^{-1}\right)$.

The quality of ccCA-CBS-2 results for this set of hydrocarbon, substituted hydrocarbon, and organic radical molecules is nearly equivalent to the G3B3 method. On the other hand, the accuracy of the ccCA methods is worse with nonhydrocarbon systems. Modifications to the ccCA may increase the accuracy of species containing second-row atoms without significantly adding to the computational cost of the ccCA methods. First, Curtiss et al. showed significant geometry differences (as large as $0.03 \AA$ ) in molecules containing second-row atoms when the DFT basis set size used in geometry optimizations was increased from $6-31 \mathrm{G}(d)$ to $6-31$ $\mathrm{G}(2 d f, p)$ in their formulation of the G3X variant. ${ }^{67}$ Second, Dunning, et $a l .{ }^{45}$ have found the addition of extra $d$ functions to the second-row correlation consistent basis sets $[\mathrm{cc}-\mathrm{pV}$ $(X+d) Z]$ to drastically improve the quality of basis set convergence and dissociation energies owing to a better description of molecular core polarization effects.

\section{B. Linear $\boldsymbol{n}$-alkanes}

The theoretical enthalpies of formation for the $n$-alkanes clearly show the basis set and size dependence of the HLC, and also accentuate the efficiency of the ccCA method compared to running large basis set coupled cluster calculations. The $\Delta H_{f}$ values obtained with various levels of theory have been recently explored by Pollack et al. ${ }^{35}$ using a composite method of CCSD(T)/CBS energies combined with the ZPE, core-valence effects, spin-orbit splitting, and relativistic corrections. Pollack et al. exceed chemical accuracy in determining enthalpies of formation for the series of $n$-alkanes from methane $\left(\mathrm{CH}_{4}\right)$ to $n$-octane $\left(\mathrm{C}_{8} \mathrm{H}_{18}\right)$, excluding heptane, via both the method of comparing atomization energies and the use of isodesmic equations. The mean absolute deviation of their seven $\Delta H_{f}$ values computed via atomization energies is only $0.23 \mathrm{kcal} \mathrm{mol}^{-1}$. Redfern, et al. ${ }^{90}$ have also studied the $n$-alkane series up to hexadecane $\left(\mathrm{C}_{16} \mathrm{H}_{34}\right)$ using variants of the $\mathrm{G} 3$ method and density functional theories. Even with the HLC, G3 and reduced-order G3 (G3[MP2/CCSD]) results showed an increasing deviation proportional to the size of the alkane system. However, most G3 methods still had computed enthalpies of formation with deviations within the range of $\pm 2 \mathrm{kcal} \mathrm{mol}^{-1}$. Their average absolute deviation from experiment for the seven straight alkanes is $0.43 \mathrm{kcal} \mathrm{mol}^{-1}$. By comparison, the increasing deviation when utilizing B3LYP 6-311+G $(3 d f, 2 p)$ is quite substantial, resulting in an average absolute deviation of $5.49 \mathrm{kcal} \mathrm{mol}^{-1}$. For larger hydrocarbons, calculation of enthalpies of formation from atomization energies creates a significant differential in the number of unpaired carbon-atom electrons com- pared to paired valence electrons. With the various $\mathrm{G} n$ implementations, this differential proportionally increases the magnitude of the HLC. Removal of the HLC is disastrous, as the error in computed $\Delta H_{f}$ values increases in the negative direction linearly and rapidly. The uncorrected G3B3 $\Delta H_{f}$ of methane has an absolute deviation of $7.3 \mathrm{kcal} \mathrm{mol}^{-1}$, increasing to an error of $20.9 \mathrm{kcal} \mathrm{mol}^{-1}$ for butane. In fact, this slope of deviation from experiment is much larger than that of standard B3LYP as the alkane chain size increases!

For the ccCA-aTZ and ccCA-CBS-1 methods, the signed deviation also becomes more negative as the molecular size increases. The ccCA-aTZ deviation of $\Delta H_{f}\left(\mathrm{CH}_{4}\right)$ is $-1.8 \mathrm{kcal} \mathrm{mol}^{-1}$, increasing in magnitude to a deviation of $-7.9 \mathrm{kcal} \mathrm{mol}^{-1}$ for hexane. With the ccCA-CBS-1 method, this increase in deviation has a smaller slope, from $-1.0 \mathrm{kcal} \mathrm{mol}^{-1}$ for methane to $-4.0 \mathrm{kcal} \mathrm{mol}^{-1}$ for octane. Again, the ccCA-CBS-2 technique is a vast improvement. For these alkane chains, the predicted enthalpy of formation is too small (giving a positive signed deviation), and the slope is smaller than all other methods with the deviation increasing from $+0.1 \mathrm{kcal} \mathrm{mol}^{-1}$ with methane to $+2.2 \mathrm{kcal} \mathrm{mol}^{-1}$ with octane. Adding the relativistic and atomic spin-orbit corrections described in Sec. IV C and IV D will act to further improve the enthalpies of formation, and the ccCA-CBS-2 mean absolute deviation of the seven straight alkane chains is $0.21 \mathrm{kcal} \mathrm{mol}^{-1}$, nearly equivalent to the composite method of Pollack et al. ${ }^{35}$ and an improvement upon the G3 variants. Figure 3 shows the deviation of $\Delta H_{f}$ with various approaches.

While the composite method in use by Pollack et al. shows impressive accuracy, few computational research groups currently have the computational resources to perform $\operatorname{CCSD}(\mathrm{T}) / \mathrm{aug}$-cc-pVQZ single-point energies for molecular systems as large or larger than $n$-octane. The tractability of running the $\operatorname{CCSD}(\mathrm{T}) / \mathrm{aug}$-cc-pVQZ octane computations (run on 1400 Itanium-2 processors for $23 \mathrm{~h}$ to compute the triples contribution to the coupled cluster energy alone $^{35}$ is questionable with mainstream technology. The most time- and disk-space consuming steps of the ccCA methods are the MP2/aug-cc-pVQZ and QCISD(T)/cc-pVTZ computations, which require significant but by no means extraordinary resources. On four SGI Origin MIPS R16000 processors, obtaining the ccCA energy of $n$-octane required nearly six days of CPU time, with the MP2/aug-cc-pVQZ step taking $45 \%$ of the CPU time, and the $\operatorname{CCSD}(\mathrm{T}) / \mathrm{cc}-$ pVTZ step taking $46 \%$ of the CPU time. On the same SGI Origin computer, the G3B3 energy of $n$-octane required 12 CPU hours. For large systems, G3B3 is likely to be an order of magnitude faster than the ccCA-CBS method. However, by including the most valuable shortcuts devised by the authors of the $\mathrm{G} n$ methods, i.e., DFT geometries and frequencies, and perturbation theory as the reference energy, the ccCA-CBS methods should provide chemical accuracy for a wide variety of systems that possess significant molecular size. 


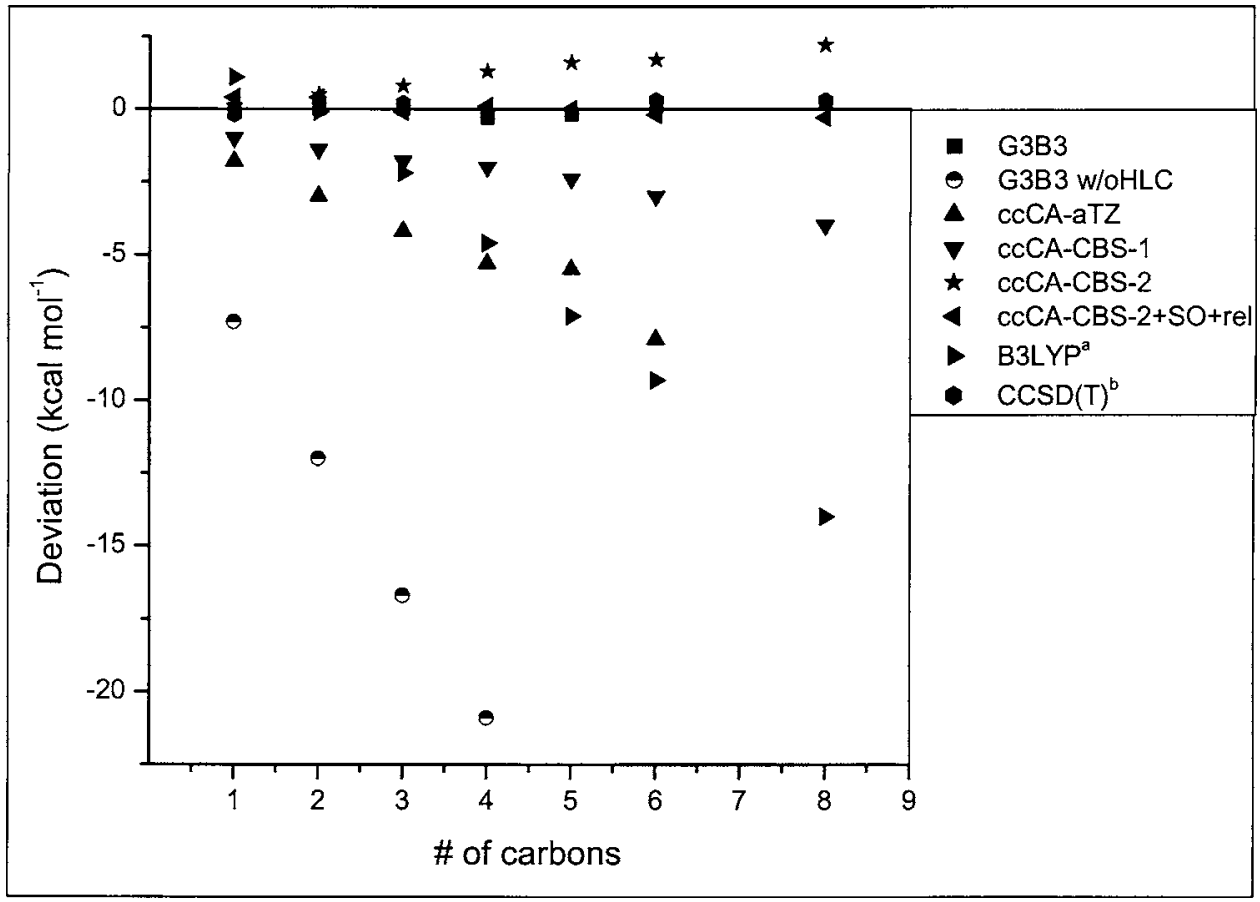

FIG. 3. A comparison of the deviation of enthalpies of formation (in $\mathrm{kcal} \mathrm{mol}^{-1}$ ) for $n$-alkanes using $\mathrm{G} n$ methods, ccCA methods, DFT, and the composite coupled cluster method of Feller and Dixon $[\operatorname{CCSD}(\mathrm{T})]$.

\section{Larger systems $\left[\mathrm{C}\left(\mathrm{NO}_{2}\right)_{4}\right.$ and adamantane]}

Lastly, we attempt to find the enthalpy of formation for two model systems where large basis set coupled cluster computations are prohibitive, tetranitromethane $\left[\mathrm{C}\left(\mathrm{NO}_{2}\right)_{4}\right]$ and adamantane $\left(\mathrm{C}_{10} \mathrm{H}_{16}\right)$. Tetranitromethane (TNM) can be used as a reagent for photonitration, for example, in syntheses of high-energy materials ${ }^{91}$ or the heterocyclization of alkenes. ${ }^{92}$ Early infrared ${ }^{93}$ and electron gas diffraction studies $^{94,95}$ showed the structure of TNM to possess $S_{4}$ point group symmetry. Theoretical studies on this molecule are scarce. The first appears to be a RHF/3-21G vibrational frequency computation supplementing a condensed phase study involving Raman spectra, ${ }^{96}$ and most recently DFT and MP2 studies, by Vladimiroff using the $6-31 \mathrm{G}(d)$ basis set. ${ }^{97}$ The ccCA geometries and harmonic frequencies obtained for this molecule should not improve on the results of the study by Vladimiroff. The enthalpy of formation for TNM at $298.15 \mathrm{~K}$ was reported in 1933 to be $19.7 \pm 0.5 \mathrm{kcal} \mathrm{mol}^{-1} .{ }^{98}$ As a molecule with more than ten nonhydrogen atoms and low Abelian point group symmetry $\left(C_{s}\right)$, TNM is a good candidate to test the ccCA methods. The ccCA-CBS- 1 and ccCA-CBS-2 methods predict a $\Delta H_{f}$ of 14.5 and $15.7 \mathrm{kcal} \mathrm{mol}^{-1}$, respectively. When atomic spin-orbit splitting is added to atomization energies, $\Delta H_{f}$ becomes 16.4 and $17.6 \mathrm{kcal} \mathrm{mol}^{-1}$. A scalar relativistic correction was obtained for $\mathrm{C}\left(\mathrm{NO}_{2}\right)_{4}$ from a frozen-core $\operatorname{CCSD}(\mathrm{T})$ wave functions using the ccpVTZ-DK (Ref. 99) basis sets and the spin-free, one-electron Douglas-Kroll-Hess (DKH) Hamiltonian. ${ }^{100-102}$ The Douglas-Kroll correction shifts the enthalpies of formation by $+2.3 \mathrm{kcal} \mathrm{mol}^{-1}$, giving final ccCA-CBS $\Delta H_{f}$ values of 18.7 and $19.9 \mathrm{kcal} \mathrm{mol}^{-1}$, respectively. The ccCA-CBS-2 enthalpy of formation for TNM is within the experimental error bars.

In terms of the number of contracted basis functions, adamantane is the largest ccCA computation to date. The largest MP2 computations were run on our SGI Origin 3900 with four MIPS R16000 processors using 16 Gbytes of RAM. The MP2/aug-cc-pVQZ computation had 1536 basis functions and took $51 \mathrm{CPU}$ hours to complete. For the ccCA methods, the limit for molecular size involves the necessary disk space required for these large MP2 computations. For example, the MP2/aug-cc-pVQZ adamantane computation used 456 Gbytes of disk space. When employing the ccCACBS-2 method with spin-orbit splitting, the $\Delta H_{f}$ value of adamantane is determined to be $-32.4 \mathrm{kcal} \mathrm{mol}^{-1}$. The ccCA-CBS-2 result is quite close to the experimental enthalpy of formation for adamantane $\left(-31.8 \mathrm{kcal} \mathrm{mol}^{-1}\right){ }^{103}$ Relativistic effects should increase the enthalpy of formation, but even with further energy corrections, the theoretical value should be at least within $2 \mathrm{kcal} \mathrm{mol}^{-1}$ of experiment. Compared to DFT methods, ccCA-CBS-2 should perform very well for large organic molecules. To contrast, the computed enthalpy of formation using $\mathrm{B} 3 \mathrm{LYP} / 6-311+\mathrm{G}(3 d 2 f, 2 p)$ energies at $\mathrm{MP} 2 / 6-31 \mathrm{G}(d)$ geometries is off by almost $33 \mathrm{kcal} \mathrm{mol}^{-1}$ from the experimental value. ${ }^{104}$ In fact, all seven of the set of DFT functionals tested in Ref. 104, some of which are relatively new, have an error in excess of $4 \mathrm{kcal} \mathrm{mol}^{-1}$ for the $\Delta H_{f}$ value of adamantane. Clearly for large molecules, DFT is not fundamentally sound for computation of theoretical $\Delta H_{f}$ values without some sort of parametrization. On the contrary, the ccCACBS-2 method can be expected to determine enthalpies of formation within $2 \mathrm{kcal} \mathrm{mol}^{-1}$ of the experimental value, even for substantially large molecules.

\section{CONCLUSIONS}

The Gaussian- $n$ "high-level correction" is primarily a basis set effect, as there is a nearly uniform bias to the deviation for enthalpies of formation. Spin-orbit splitting and relativistic effects further offset these quantities in the 
"wrong" direction, i.e., further from experiment. This results in a HLC that must not only be reparametrized for the various deficiencies in the one- and $n$-particle treatment of the electronic Schrödinger equation but also for smaller additive physical corrections. Upon increasing size of even wellbehaved single-reference molecular systems, basis set deficiencies in the $\mathrm{G} n$ model chemistries become exacerbated relative to the component atoms of the molecule; thus the quality of computing molecular properties that compare total energies of molecules to the energies of their component atoms will deteriorate rapidly without inclusion of a HLC.

If optimized parameters such as the HLC are avoided, the accuracy of theoretical electron affinities and enthalpies of formation will improve as the reference basis set used in the composite method is enlarged. When MP2 energies obtained with the correlation consistent one-particle basis sets are extrapolated to the CBS limit as in the ccCA-CBS-1 and ccCA-CBS-2 methods, then ionization potentials, electron affinities, and proton affinities compare satisfactorily to experiment. On the other hand, the initial accuracy of enthalpies of formation is modest, but with the ccCA-CBS-2 method, the error is biased in the favorable direction such that additive corrections improve the mean average deviation. When atomic spin-orbit and approximate scalar relativistic effects are included in the total energies, the ccCACBS-2 average absolute deviations are $0.81 \mathrm{kcal} \mathrm{mol}^{-1}$ for 48 enthalpies of formation, $0.80 \mathrm{kcal} \mathrm{mol}^{-1}$ for 38 ionization potentials, $1.09 \mathrm{kcal} \mathrm{mol}^{-1}$ for 25 electron affinities, and $1.55 \mathrm{kcal} \mathrm{mol}^{-1}$ for 8 proton affinities. In total for the G2-1 test set, excluding the seven enthalpies of formation for silicon-containing compounds, the ccCA-CBS-2 method with relativistic and spin-orbit splitting corrections has an average absolute deviation of $0.92 \mathrm{kcal} \mathrm{mol}^{-1}$. For larger organic systems, the ccCA-CBS-2 method gives the results comparable and sometimes better than those obtained with G3B3.

Enthalpies of formation for two large molecules, $\mathrm{C}\left(\mathrm{NO}_{2}\right)_{4}$ with 13 heavy atoms and low point group symmetry and adamantane with 26 total atoms, are computed to be within $2 \mathrm{kcal} \mathrm{mol}^{-1}$ of experimental estimates. While a composite approach free of empirical parameters (i.e., the HLC) is of great utility, the main drawback is the higher computational cost requirements. In terms of CPU time, the computational cost of the ccCA-CBS methods are at least an order of magnitude larger than that of the G3 methods. For the ccCA methods, the primary technological limitation of tractable molecular size is disk space. Our MP2/aug-cc-pVQZ adamantane computation required almost 500 Gbytes of disk, which is a large but not extraordinary amount. Therefore, employing the ccCA-CBS methods to compute energies of molecules substantially larger than adamantane is currently possible. Computing ccCA-CBS-2 energies for medium-sized systems is reasonable with typical computational resources.

The ccCA approach provides a "black box" means to obtain the atomic and molecular energies, resulting in nearchemical accuracy for energy-related properties of most systems studied to date. Further systematic improvement planned for the approach includes modifications of second- row basis sets, imposing state averaging on open-shell atomic computations, improving geometry optimizations and electron correlation treatments, and employing a more systematic treatment of scalar relativistic effects. All of these modifications should systematically improve the accuracy of molecular energies, especially on molecules containing second-row atoms, with little sacrifice in computational cost. These modifications are currently being explored with the larger and more diverse G3/99 training set.

\section{ACKNOWLEDGMENTS}

CASCaM is supported by a grant from the United States Department of Education. This research is partially supported by a grant from the United States Department of Energy to one of the authors (T.R.C.) Grant No. DE-FG0203ER 15387 and by a National Science Foundation CAREER Award No. CHE-0239555 to another author (A.K.W.). Computational resources were provided via the National Science Foundation (CHE-0342824), and by the National Computational Science Alliance under CHE010021 and utilized the NCSA IBM p690. Additional support was also provided by the University of North Texas Academic Computing Services for the use of the UNT Research Cluster. Professor Kirk A. Peterson (Department of Chemistry, Washington State University) is thanked for providing new Group IA and IIA correlation consistent basis sets prior to their publication.

${ }^{1}$ J. A. Pople, B. T. Luke, M. J. Frisch, and J. S. Binkley, J. Chem. Phys. 89, 2198 (1985).

${ }^{2}$ J. A. Pople, M. Head-Gordon, D. J. Fox, K. Raghavachari, and L. A. Curtiss, J. Chem. Phys. 90, 5622 (1989).

${ }^{3}$ L. A. Curtiss, C. Jones, G. W. Trucks, K. Raghavachari, and J. A. Pople, J. Chem. Phys. 93, 2537 (1990).

${ }^{4}$ L. A. Curtiss, K. Raghavachari, G. W. Trucks, and J. A. Pople, J. Chem. Phys. 94, 7221 (1991).

${ }^{5}$ L. A. Curtiss, K. Raghavachari, P. C. Redfern, V. Rassolov, and J. A. Pople, J. Chem. Phys. 109, 7764 (1998).

${ }^{6}$ L. A. Curtiss, K. Raghavachari, P. C. Redfern, and J. A. Pople, J. Chem. Phys. 106, 1063 (1997).

${ }^{7}$ K. Raghavachari, B. B. Stefanov, and L. A. Curtiss, J. Chem. Phys. 106, 6764 (1997)

${ }^{8}$ L. A. Curtiss, K. Raghavachari, P. C. Redfern, and J. A. Pople, J. Chem. Phys. 112, 7374 (2000).

${ }^{9}$ K. Raghavachari and L. A. Curtiss, in Modern Electronic Structure Theory, edited by D. R. Yarkony (World Scientific, Singapore, 1995), Vol. II, p. 991.

${ }^{10}$ L. A. Curtiss, P. C. Redfern, and D. J. Frurip, in Reviews of Computational Chemistry, edited by K. B. Lipkowitz and D. B. Boyd (WileyVCH, New York, 2000), Vol. 15, p. 147.

${ }^{11}$ G. A. Petersson and M. J. Frisch, J. Phys. Chem. A 104, 2183 (2000).

${ }^{12}$ J. A. Montgomery, M. J. Frisch, J. W. Ochterski, and G. A. Petersson, J. Chem. Phys. 110, 2822 (1999).

${ }^{13}$ G. A. Petersson, Abstr. Pap. - Am. Chem. Soc. 212, 175 (1996).

${ }^{14}$ J. W. Ochterski, G. A. Petersson, and J. A. Montgomery, J. Chem. Phys. 104, 2598 (1996).

${ }^{15}$ A. L. L. East and W. D. Allen, J. Chem. Phys. 99, 4638 (1993).

${ }^{16}$ J. P. Kenny, W. D. Allen, and H. F. Schaefer, J. Chem. Phys. 118, 7353 (2003).

${ }^{17}$ J. M. Gonzales, C. Pak, R. S. Cox, W. D. Allen, H. F. Schaefer, A. G. Császár, and G. Tarczay, Chem.-Eur. J. 9, 2173 (2003).

${ }^{18}$ A. G. Császár, W. D. Allen, and H. F. Schaefer, J. Chem. Phys. 108, 9751 (1998)

${ }^{19}$ A. G. Császár, W. D. Allen, Y. Yamaguchi, and H. F. Schaefer, in Computational Molecular Spectroscopy, edited by P. Jensen and P. R. Bunker (Wiley, New York, 2000), p. 15.

${ }^{20}$ A. G. Császár, P. G. Szalay, and M. L. Leininger, Mol. Phys. 100, 3879 (2002). 
${ }^{21}$ A. G. Császár, M. L. Leininger, and A. Burcat, J. Phys. Chem. A 107, 2061 (2003).

${ }^{22}$ A. G. Császár, M. L. Leininger, and V. Szalay, J. Chem. Phys. 118, 10631 (2003).

${ }^{23}$ S. Parthiban and J. M. L. Martin, J. Chem. Phys. 114, 6014 (2001).

${ }^{24}$ J. M. L. Martin and G. de Oliveira, J. Chem. Phys. 111, 1843 (1999).

${ }^{25}$ P. G. Szalay, A. Tajti, and J. F. Stanton, Mol. Phys. 103, 2159 (2005).

${ }^{26}$ A. Tajti, P. G. Szalay, A. G. Császár, M. Kallay, J. Gauss, E. F. Valeev, B. A. Flowers, J. Vazquez, and J. F. Stanton, J. Chem. Phys. 121, 11599 (2004).

${ }^{27}$ D. Feller and D. A. Dixon, J. Phys. Chem. A 107, 9641 (2003).

${ }^{28}$ D. Feller, D. A. Dixon, and J. S. Francisco, J. Phys. Chem. A 107, 1604 (2003).

${ }^{29}$ D. Feller, K. A. Peterson, W. A. de Jong, and D. A. Dixon, J. Chem. Phys. 118, 3510 (2003).

${ }^{30}$ D. A. Dixon, D. Feller, and J. S. Francisco, J. Phys. Chem. A 107, 186 (2003).

${ }^{31}$ D. A. Dixon, D. Feller, and K. A. Peterson, J. Chem. Phys. 115, 2576 (2001).

${ }^{32}$ D. Feller and D. A. Dixon, J. Phys. Chem. A 104, 3048 (2000).

${ }^{33}$ D. A. Dixon, D. Feller, and G. Sandrone, J. Phys. Chem. A 103, 4744 (1999).

${ }^{34}$ D. Feller and D. A. Dixon, J. Phys. Chem. A 103, 6413 (1999).

${ }^{35}$ L. Pollack, T. L. Windus, W. A. de Jong, and D. A. Dixon, J. Phys. Chem. A 109, 6934 (2005).

${ }^{36}$ D. Feller and K. A. Peterson, J. Chem. Phys. 108, 154 (1998).

${ }^{37}$ D. Feller and K. A. Peterson, J. Chem. Phys. 110, 8384 (1999).

${ }^{38}$ P. L. Fast, J. Corchado, M. L. Sanchez, and D. G. Truhlar, J. Phys. Chem. A 103, 3139 (1999)

${ }^{39}$ P. L. Fast, J. C. Corchado, M. L. Sanchez, and D. G. Truhlar, J. Phys. Chem. A 103, 5129 (1999).

${ }^{40}$ P. L. Fast and D. G. Truhlar, J. Phys. Chem. A 104, 6111 (2000).

${ }^{41}$ J. Cioslowski, M. Schimeczek, G. Liu, and V. Stoyanov, J. Chem. Phys. 113, 9377 (2000)

${ }^{42}$ S. Grimme, J. Phys. Chem. A 109, 3067 (2005).

${ }^{43}$ T. H. Dunning, Jr., J. Chem. Phys. 90, 1007 (1989).

${ }^{44}$ S. S. Xantheas and T. H. Dunning, Jr., J. Phys. Chem. 97, 18 (1993).

${ }^{45}$ T. H. Dunning, Jr., K. A. Peterson, and A. K. Wilson, J. Chem. Phys. 114, 9244 (2001).

${ }^{46}$ A. K. Wilson, D. E. Woon, K. A. Peterson, and T. H. Dunning, Jr., J. Chem. Phys. 110, 7667 (1999).

${ }^{47}$ A. K. Wilson, D. E. Woon, K. A. Peterson, and T. H. Dunning, Jr., Abstr. Pap.-Am. Chem. Soc. 213, 60 (1997).

${ }^{48}$ R. A. Kendall, T. H. Dunning, Jr., and R. J. Harrison, J. Chem. Phys. 96 6796 (1992)

${ }^{49}$ D. E. Woon and T. H. Dunning, Jr., J. Chem. Phys. 100, 2975 (1994).

${ }^{50}$ D. E. Woon and T. H. Dunning, Jr., J. Chem. Phys. 103, 4572 (1995).

${ }^{51}$ K. A. Peterson and T. H. Dunning, Jr., J. Chem. Phys. 117, 10548 (2002).

${ }^{52}$ N. B. Balabanov and K. A. Peterson, J. Chem. Phys. 123, 064107 (2005).

${ }^{53}$ M. Head-Gordon, J. A. Pople, and M. J. Frisch, Chem. Phys. Lett. 153, 503 (1988).

${ }^{54}$ M. J. Frisch, M. Head-Gordon, and J. A. Pople, Chem. Phys. Lett. 166, 281 (1990).

${ }^{55}$ P. C. Hariharan and J. A. Pople, Theor. Chim. Acta 28, 213 (1973).

${ }^{56}$ R. Krishnan, M. J. Frisch, and J. A. Pople, J. Chem. Phys. 72, 4244 (1980).

${ }^{57}$ M. J. Frisch, J. A. Pople, and J. S. Binkley, J. Chem. Phys. 80, 3265 (1984).

${ }^{58}$ T. Clark, J. Chandrasekhar, G. W. Spitznagel, and P. v. R. Schleyer, J. Comput. Chem. 4, 294 (1983).

${ }^{59}$ J. A. Pople, M. Head-Gordon, and K. Raghavachari, J. Chem. Phys. 87, 5968 (1987)

${ }^{60}$ C. E. Moore, Atomic Energy Levels, Natl. Bur. Stand. (U.S.) 467 (U.S. Department of Commerce, Washington D.C., 1952).

${ }^{61}$ L. A. Curtiss, J. E. Carpenter, K. Raghavachari, and J. A. Pople, J. Chem. Phys. 96, 9030 (1992).

${ }^{62}$ L. A. Curtiss, P. C. Redfern, B. J. Smith, and L. Radom, J. Chem. Phys. 104, 5148 (1996).

${ }^{63}$ L. A. Curtiss, K. Raghavachari, P. C. Redfern, A. G. Baboul, and J. A. Pople, Chem. Phys. Lett. 314, 101 (1999).
${ }^{64}$ L. A. Curtiss, K. Raghavachari, and J. A. Pople, J. Chem. Phys. 98, 1293 (1993).

${ }^{65}$ L. A. Curtiss, P. C. Redfern, K. Raghavachari, V. Rassolov, and J. A. Pople, J. Chem. Phys. 110, 4703 (1999).

${ }^{66}$ L. A. Curtiss, K. Raghavachari, P. C. Redfern, and J. A. Pople, J. Chem. Phys. 112, 1125 (2000).

${ }^{67}$ L. A. Curtiss, P. C. Redfern, K. Raghavachari, and J. A. Pople, J. Chem. Phys. 114, 108 (2001).

${ }^{68}$ A. G. Baboul, L. A. Curtiss, P. C. Redfern, and K. Raghavachari, J. Chem. Phys. 110, 7650 (1999).

${ }^{69}$ C. W. Bauschlicher, Jr., and H. Partridge, J. Chem. Phys. 103, 1788 (1995).

${ }^{70}$ A. T. Scott and L. Radom, J. Phys. Chem. 100, 16502 (1996).

${ }^{71}$ L. A. Curtiss and K. Raghavachari, Theor. Chem. Acc. 108, 61 (2002).

${ }^{72}$ D. Feller, J. Chem. Phys. 96, 6104 (1992).

${ }^{73}$ D. Feller, J. Chem. Phys. 98, 7059 (1993).

${ }^{74}$ K. A. Peterson, D. E. Woon, and T. H. Dunning, Jr., J. Chem. Phys. 100, 7410 (1994).

${ }^{75}$ M. J. Frisch, G. W. Trucks, H. B. Schlegel et al., Gaussian 03, Revision C.02 (Gaussian, Inc., Wallingford, CT, 2004).

${ }^{76}$ R. D. Amos, A. Bernhardsson, A. Berning et al., MOLPRO, a package of $a b$ initio programs designed by H.-J. Werner and P. J. Knowles, version 2002.6 (University of Birmingham, Birmingham, UK, 2002).

${ }^{77}$ K. A. Peterson, D. E. Woon, and T. H. Dunning, Jr. (unpublished).

${ }^{78}$ R. S. Grev and H. F. Schaefer, J. Chem. Phys. 97, 8389 (1992).

${ }^{79}$ C. L. Collins and R. S. Grev, J. Chem. Phys. 108, 5465 (1998).

${ }^{80}$ O. Visser, L. Visscher, P. J. C. Aerts, and W. C. Nieuwpoort, Theor. Chem. Acc. 81, 405 (1992).

${ }^{81}$ A. Ricca and C. W. Bauschlicher, J. Phys. Chem. A 102, 876 (1998).

${ }^{82}$ J. M. W. Chase, NIST-JANAF Tables (4th ed.); J. Phys. Chem. Ref. Data Monogr. 9, 1 (1998).

${ }^{83}$ R. M. Lucente-Shultz, B. P. Prascher, N. J. DeYonker, and A. K. Wilson (unpublished).

${ }^{84}$ G. S. Kedziora, J. A. Pople, V. A. Rassolov, M. A. Ratner, P. C. Redfern, and L. A. Curtiss, J. Chem. Phys. 110, 7123 (1999).

${ }^{85}$ L. A. Curtiss, P. C. Redfern, V. Rassolov, G. Kedziora, and J. A. Pople, J. Chem. Phys. 114, 9287 (2001).

${ }^{86}$ G. S. Kedziora, J. A. Pople, M. A. Ratner, P. C. Redfern, and L. A. Curtiss, J. Chem. Phys. 115, 718 (2001).

${ }^{87}$ W. Kutzelnigg, E. Ottschofski, and R. Franke, J. Chem. Phys. 102, 1740 (1995)

${ }^{88}$ L. A. Curtiss, K. Raghavachari, P. C. Redfern, G. S. Kedziora, and J. A. Pople, J. Phys. Chem. A 105, 227 (2001).

${ }^{89}$ B. Ruscic, J. E. Boggs, A. Burcat et al., J. Phys. Chem. Ref. Data 34, 573 (2005).

${ }^{90}$ P. C. Redfern, P. Zapol, L. A. Curtiss, and K. Raghavachari, J. Phys. Chem. A 104, 5850 (2000).

${ }^{91}$ V. Fargeas, F. Favresse, D. Mathieu, I. Beaudet, P. Charrue, B. Lebret, M. Piteau, and J. P. Quintard, Eur. J. Org. Chem. 2003, 1711 (2003).

${ }^{92}$ E. B. Averina, E. M. Budynina, O. A. Ivanova, Y. K. Grishin, S. M. Gerdov, T. S. Kuznetsova, and N. S. Zefirov, Russ. J. Org. Chem. 40, 162 (2004).

${ }^{93}$ P. H. Lindenmeyer and P. M. Harris, J. Chem. Phys. 21, 408 (1953).

${ }^{94}$ A. J. Stosick, J. Am. Chem. Soc. 61, 1127 (1939).

${ }^{95}$ N. I. Sadova, N. I. Popik, and L. V. Vilkov, J. Mol. Struct. 31, 399 (1976).

${ }^{96}$ J. F. Arenas, J. C. Otero, and J. Soto, J. Mol. Struct. 298, 191 (1993).

${ }^{97}$ T. Vladimiroff, J. Mol. Struct.: THEOCHEM 452, 233 (1998).

${ }^{98}$ V. P. Lebedev, E. A. Miroschnichenko, Y. N. Matyushin, V. P. Larionov, V. S. Romanov, Y. E. Bukolov, G. M. Denisov, A. A. Balepin, and Y. A. Lebedev, Russ. J. Phys. Chem. 49, 1133 (1975).

${ }^{99}$ W. A. de Jong, R. J. Harrison, and D. A. Dixon, J. Chem. Phys. 114, 48 (2001).

${ }^{100}$ B. A. Hess, Phys. Rev. A 33, 3742 (1986)

${ }^{101}$ B. A. Hess, Phys. Rev. A 32, 756 (1985).

${ }^{102}$ M. Douglas and N. M. Kroll, Ann. Phys. (N.Y.) 82, 89 (1974).

${ }^{103}$ T. Clark, T. Mc, O. Knox, M. A. McKervey, H. Mackle, and J. J. Rooney, J. Am. Chem. Soc. 101, 2404 (1979).

${ }^{104}$ L. A. Curtiss, P. C. Redfern, and K. Raghavachari, J. Chem. Phys. 123, 124017 (2005). 